\title{
Ueber die Behandlung der Psoriasis mit grossen Dosen von Jodkalium, nebst Be- merkungen über die Jodwirkung.
}

\author{
Von \\ Dr. Seifert, \\ Privatdocent in Würzburg.
}

Die Psoriasis gehört zu jenen Krankheiten der Haut, über deren Wesen wir trotz sorgfältiger Untersuchungen noch wenig Sicheres wissen. So lange wir eine Einsicht in das Wesen dieses Krankheitsprocesses nicht gewinnen, wird uuser therapeutisches Handeln noch kein zielbewusstes sein können und auch noch die eine Hauptfrage unerledigt bleiben, ob wir mehr von einer externen oder mehr ron einer internen Behandlung erwarten können. Es steht ja ausser Zweifel, dass durch eine ganze Reihe ron externen Behandlungsmethoden: Chrysarobin, Pyrogallussäure, Naphthol, Anthrarobin, Hydracetin, Hydroxylamin, Aristol, Europhen, Gallanol, Gallactophenin etc. Psoriasis geheilt werden kann, aber eine solche Heilung stellt immer nur eine temporäre dar, früher oder später kommen Recidive, ohne dass wir die Macht besitzen, sie hintanzuhalten. Einzelne dieser stärker wirkenden Medicamente wie Chrysarobin, Pyrogallussäure, Hydroxylamin zeichnen sich auch noch unvortheilhaft aus durch ihre unangenehmen und nicht immer ungefährlichen Nebenwirkungen. Unter solchen Umständen ist es nicht zu verwundern, dass ausser der externen Behandlungsmethode auch immer wieder das eine oder andere Mittel für den internen Gebrauch empfohlen wird. Zu jenen Mitteln, welche sich am 
längsten behauptet haben, gehört der Arsenik und das Jodkalium. Wir haben auf unserer Abtheilung früher vielfach Gebrauch gemacht rom Arsenik mit wechselndem Erfolge; zu der Zeit, als ich die Syphilido-Klinik übernahm (1887), waren dieselben so wenig befriedigend, dass ich mit Freuden die Empfehlung des Jodkaliums durch $\mathrm{Haslund}^{1}$ ) begrüsste und sogleich eine Reihe von Psoriasisfällen nach der von ihm vorgeschlagenen Methode behandelte. Aus der Arbeit von Ha sl und geht hervor, dass die erste Empfehlung des Jodkaliums bei Psoriasis zuriuckführen ist auf $\left(\mathrm{Greves} \mathrm{s}^{2}\right.$ ), der sowohl bei Erwachsenen als bei Kindern gute Erfolge von dessen Anwendung sah, wenn es in allmälig steigender Dosis gegeben wurde. Wenn der Patient bis zu $4 \mathrm{Gr}$. in täglicher Dosis gelangt war, fing das Mittel an, seine günstige Wirkung auf die Hautkrankheit zu zeigen, aber erst bei täglicher Dosis von $10 \mathrm{Gr}$. ging es rapid besser. Er hat beobachtet, dass zuerst Kopf und Hals, dann Truncus und zuletzt die Extremitäten glatt werden. Eine schädliche Wirkung der grösseren Dosen Jodkaliums will er nie beobachtet haben.

Boeck ${ }^{3}$ ) fügt dem Referate über diesen Artikel einen Fall aus seiner eigenen Praxis hinzu, ein 5jähriges Mädchen betreffend, das an einer fast universellen Psoriasis litt; nach Gebrauch von 79 Gr. Jodkalium war die Krankheit in gutem Rückgange.

Haslund ${ }^{4}$ ) berichtet nun über eine stattliche Anzahl von Fällen, welche mit Jodkalium in steigender Dosis behandelt wurden. Unter 50 Fällen wurde in 46 Fällen vällige Genesung durch die Cur erreicht, während 4 Fälle bedeutende Besserung zeigten, in 6 Fällen ist kein Resultat erzielt worden, indem die Psoriasis bei dem Abschluss der Cur ganz unverändert geblieben ist, oder sich reichliche neue Efflorescenzen zeigten zur selben Zeit, wo die alten fast verschwunden waren. Die Dauer der Behandlung war sehr verschieden, im Durchschnitt wenig mehr als 7 Wochen. Dem entsprechend war die ganze Menge von Jodkalium, welche gebraucht wurde, um die Krankheit zu heilen, sehr verschieden gross. Was die Männer betrifft, so variirte sie von 160-1390 Gr., was die Frauen angeht, von 526-1328 Gr. und für die Kinder von 277 bis 1520 Gr. In den meisten Fällen sah man, dass, wenn die Krankeit einmal zu schwinden angefangen hatte, es dann nur kurze Zeit dauerte, bis die Haut glatt war; in einzelnen Fällen fand dieser Rückgang, wenn er

\footnotetext{
1) Vierteljahrsschrift f. Dermat. u. Syph. 1887.

$\left.{ }^{2}\right)$ Tidsskrift for prakt. Medic. 16. 1881 .

3) Vierteljahrsschrift f. Dermat. 1882. 3.

$\left.{ }^{4}\right)$ 1. c.
} 
Ueber die Behandl. der Psoriasis mit grossen Dosen von JK. 325

begonnen, so rapid statt, dass eine sehr ausgebreitete Efflorescenz im Laufe einer Woche oder in noch kürzerer Zeit fast gänzlich verschwunden war.

Das Jodkali wurde in der Weise verabreicht, dass mit einer Lösung von $10 \mathrm{Gr}$. Jodkali auf $200 \mathrm{Gr}$. Wasser angefangen wurde, wovon der Patient 1 Esslöffel voll 4 mal täglich bekam. Bei kleinen Kindern wurde mit 5 Gr. auf $100 \mathrm{Gr}$. angefangen. Nach 2-3 Tagen wurden 6 Essslöffel täglich genommen, nach weiteren 2 Tagen 8 Löffel und so fort, bis der Patient 12 Löffel erreichte, was also die ganze Portion ausmachte. Hatte der Patient ein paar Tage hindurch die ganze Portion eingenommen, so wurde die folgende Portion zu 12 : 200 verschrieben und so fort, alle 2-3 Tage die Auflösung 2 Gr. stärker als die frühere genommen. Für reichliche Wasserzufuhr besonders nach den stärkeren Dosen wurde gesorgt. $\mathrm{Hasl}$ und hat einem seiner. Patienten bis zu 50 Gr. p. die gegeben.

Es ist Haslund aufgefallen, dass die Patienten so grosse Dosen gut vertragen konnten, nur in dem einen Fall, der bis zu $50 \mathrm{Gr}$. täglich gestiegen war, handelte sich um ernstlichere Erscheinungen von Jodismus, bestehend in einer gewissen Abgestumpftheit, der Unfähigkeit die Gedanken zu sammeln, Verwirrtheit, Kopfweh, Ohrensausen, Herzklopfen. Am folgenden Morgen war der Herzschlag ziemlich tumultuarisch und die Töne unrein, aber bei der Untersuchung wurde sonst nichts Abnormes beobachtet, kein Steigen noch Fallen der Temperatur. Diese Erscheinungen von Herzparese gingen bald vorüber.

Absolute Idiosynkrasie gegen Jodkalium hat Haslund nur einige Male beobachtet.

Die meisten der Patienten hatten nach überstandener Krankheit an Gewicht zugenommen und für mehrere, welche an Gewicht abgenommen hatten, waren besondere Verhältnisse geltend zu machen, die einen solchen Verlust erklären konnten.

Aus seinen Beobachtungen zieht $\mathrm{H}$ aslund den Schluss, dass wir im Jodkalium in grossen Dosen gegeben, ein Mittel besitzen, das mit ziemlicher Sicherheit vermag, einen Ausbruch der Psoriasis zu heilen, ferner, dass wir gegenwärtig kein Mittel kennen, das, imneriich gebraucht, in so kurzer Zeit, wie dieses heilend wirki. Ueber das Auftreten von Recidiven konnten bestimmte Angaben nicht gemacht werden, weil über das weitere Schicksal des grösseren Theiles der Fälle jede Nachricht fehlt. Ueber die Weise, wie das Jodkalium wirkt und heilend wirkt, haben die Versuche von $\mathrm{H}$ a slund nicht Licht schaffen können.

Von Interesse sind noch die Beobachtungen, welche Haslund über das Verhalten des Pulses gemacht hat. Nur einige Male zeigte sich ganz vorübergehend eine leichte Unregelmässigkeit des Pulses. Die Schnelligkeit des Pulses wurde stets durch die grossen Dosen von Jodkalium vermehrt. In den ersten 10-14 Tagen stieg der Puls gewöhnlich bis 100, ging dann langsam hinauf bis auf 130, bei Einzelnen sogar bis 140 Schläge in der Minute. Von einer Einwirkung auf die Körpertemperatur ist in dem Aufsatze nirgends die Rede. 
Molèsnes ${ }^{1}$ ) hat Jodkalium bei Psoriasis ebenfalls angewandt, zum Theil in noch grösseren Dosen als Haslund.

Auf der dermatologischen Klinik in Breslau sind eine ganze Reihe von Psoriasisfällen mit Jodkalium behandelt worden (s. Bienstack ${ }^{2}$ ) Es hat sich ergeben, dass Dosen von $27 \mathrm{Gr}$. Jodkalium pro die mit reichlich Milch gegeben, ohne Nachtheil vertragen wurden, dass aber eine wirkliche Heilwirkung in keinem einzigen der Fälle constatirt werden konnte.

Doutrelepont $\left.{ }^{3}\right)$ stellte in der niederrheinischen Gesellschaft in Bonn eine Patientin vor, die von ihrer universellen Psoriasis durch Jodkalium geheilt worden war. Die Patientin hatte in 102 Tagen allmälig $1920 \mathrm{Gr}$. Jodkalium eingenommen, die grösste Tagesdosis betrug $28 \mathrm{Gr}$. Die Patientin hat diese grosse Dosen sehr gut vertragen und sich während des Aufenthaltes in der Klinik sehr gekräftigt. Ausser diesem Falle sind in der Poliklinik noch 2 mit Psoriasis behaftete Patienten mit grossen Dosen Jodkali behandelt und geheilt worden.

Barduzzi ${ }^{4}$ ) sah in 3 Fällen von inveterirter Psoriasis vom inneren Gebrauch des Jodkali sehr guten Erfolg. Er liess die Cur mit 1.0 pro die beginnen und jeden 5. Tag um $1 \mathrm{Gr}$. steigen, bis die tägliche Dosis von $7 \mathrm{Gr}$. erreicht war. $\mathrm{Zu}$ so heroischen Dosen, wie Haslund sie anwendet, stieg Barduzzi nie, er hält solche auch nicht für nothwendig, da er bereits mit der Maximaldosis von $7 \mathrm{Gr}$. eine auffallende und andauernde Besserung der Psoriasis erzielte.

Gutteling ${ }^{5}$ ) berichtet über 22 Fälle von. Psoriasis, welche in der Klinik und Poliklinik von van Haren Noman zu Amsterdam mit Jodkali behandelt wurden. Von diesen Fällen wurden 5 völlig geheilt (1 mal nachdem ein Recidiv eingetreten), 5 zeigten nur noch spärliche Reste, als sie sich der Behandlung entzogen, 5 sind fast ganz geheilt und 7 sind gebessert, doch konnte Heilung nicht erzielt werden, zum Theil wegen beträchtlichem Jodismus. Im Allgemeinen wurde Jodkali auch in grösseren Dosen gut ertragen. Die Patienten nahmen an Körpergewicht zu. Albuminurie wurde nicht beobachtet. Die Pulsfrequenz war bis 100 und 120 erhöht. Es wurde das Jodkalium bis zu Tagesdosen von $30-40$ ja $57 \mathrm{Gr}$. und in Totaldosen von $2 \cdot 3$ und $3 \cdot 7 \mathrm{Kg}$. gegeben. Die Erfolge haben demnach den Verfasser nicht befriedigt, es scheinen ihm nur die recenten Fälle für das Jodkalium geeignet zu sein.

Fabry ${ }^{6}$ ) theilt in seinem Aufsatz ,zur Behandlung der Psoriasis, insbesondere mit Hydroxylaminum muriaticum" mit, dass in der Klinik

1) Virch.-Hirsch. 1889.

$\left.{ }^{2}\right)$ Zur Therapie der Psoriasis. Dissert. Breslau. 1888.

3) Berl. klin. Wochenschr. 14. 1888.

4) Gazz. degli ospitali. 17. 1889.

5) Nederl. Tijdschr. v. Geneeslunde. 17. 1889.

6) Vierteljahrschr. f. Dermat. u. Syphil. 1889. 2. 
Ueber die Behandl. der Psoriasis mit grossen Dosen von JK. 327

von Doutrelepont bei einer Reihe von Psoriasiskranken Jodkalium in kleinen Dosen (von einer 5\% Lösung zwei bis sechs Esslöffel pro die) als die örtliche Medication unterstützendes Heilmittel verabreicht wurde.

Shomackerl) empfiehlt auser verschiedenen anderen Mitteln auch Jodkali, doch ist aus dem Referat über diesen Aufsatz nicht ersichtlich, nach welcher Vorschrift und mit welchem Erfolg das Mittel gegeben wurde.

L ucian $i^{2}$ ) behandelte einen 53jährigen Mann, der schon seit seinem 17. Lebensjabre an Psoriasis litt, welche allen Heilungsversuchen widerstanden hatte, mit Jodkali in steigenden Dosen von 3 bis 15 Gr. pro die. Schon nach 20 Tagen war eine wesentliche Besserung erzielt worden. Erscheinungen von Jodismus wurden nicht beobachtet, ebensowenig gastrische Störungen. Da neben dem innerlichen Gebrauche des Jodkalium auch eine örtliche Behandlung (Creolin- und Ichthyolsalbe) eingeleitet wurde, dürfte im vorliegenden Falle die Involution der Psoriasis nur zum Theil auf Rechnung des Jodkali zu setzen sein.

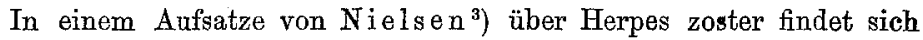
als gelegentliche Bemerkung, dass in dem Communehospital in Kopenhagen eine grosse Anzahl von Psoriasisfällen mit Jodkalium in grossen Dosen behandelt worden sei.

Polotebnoff ${ }^{4}$ ) hat nur in einem Falle von Psoriasis grosse Dosen Jodkali gegeben, allerdings ohne Erfolg.

Gamberinis) sah rom Jodkali in grossen Dosen keine Wirkung.

Kaposi $i^{\theta}$ kann dem Jodkali, wenn es consequent gegeben wird, eine Wirksamkeit auf die Psoriasis nicht absprechen. innerlich.

Ma pother ${ }^{7}$ empfiehlt ausser Quecksilber äusserlich auch Protojodid

Norton ${ }^{8}$ ) gibt von seiner Jodwasserstoffsäure-Mixtur 1-2 Theelöffel voll 3 Mal täglich bei Psoriasis. 1 Theelöffel roll des Syrups entspricht 0.3 Jodkali. Der Aufsatz von de Molènes ${ }^{9}$ ) über die Wirkung des Jodkalium in grossen Dosen auf den Organismus und seine Anwendung bei Psoriasis ist mir nicht zugänglich gewesen.

1) The med. Register. Philadelphia. 10. Nov. 1888.

$\left.{ }^{2}\right)$ Contrib. alla cura della psoriasi con forti dosi di joduro di potassio. Lo speriment. 4. 1889.

$\left.{ }^{3}\right)$ Ueber das Auftreten von Herpes zoster während Arsenikbehandlung. Monatsh. f. prakt. Dermatol. XI. 1890.

4) Monatsh. f. prakt. Dermat. Ergänzungsheft L. 1891.

5) Giorn. ital. d. mal. vener. e d. pelle. IV. 1892.

6) Lehrb. II. Aufl.

7 The brit. med. Journ. 1891.

$\left.{ }^{8}\right)$ The med. and surg. Report. 20. 1890.

9) Arch. génér. de méd. Juin 1889. 
Dubois-Habenith ) ist der Ansicht, dass ebensowenig wie Arsenik so auch Jodkalium ein Specificum gegen Psoriasis sei.

Auch Wolff ${ }^{2}$ ) scheint in einzelnen Fällen von Psoriasis grosse Dosen Jodkalium zu geben, wie man aus einer Bemerkung in seinem. Vortrag über Syphilis hereditaria tarda schliessen kann.

In Betreff der Jodkaliumtherapie macht Hillebrand ${ }^{3}$ ) darauf aufmerksam, dass bei Kindern und alten Leuten in Bezug auf die Dosis etwas grössere Vorsicht erforderlich sei als bei Erwacbsenen.

Lang ${ }^{4}$ ) hat einem Psoriasis-Kranken grosse Dosen Jodkalium gegeben, doch findet sich in der im Archiv mitgetheilten Notiz nichts über die Wirkung dieces Mittels auf die Psoriasis angegeben, der Patient hatte ausser der Psoriasis eine Geschwulst im linken Hoden, die anfangs für Syphilis gehalten werden musste, nach der Exstirpation sich aber als Sarkom erwies.

Nach Crocker ${ }^{5}$ ) beeinflusst $J K$ in sehr grossen Dosen günstig die Psoriasis.

Aus dieser Zusammenstellung geht hervor, dass im Allgemeinen die Jodtherapie der Psoriasis wenig Anhänger gefunden hat, so dass es von Interesse sein wird, von den Erfahrungen Mittheilung zu machen, die wir mit der Jodtherapie auf der Abtheilung für Hautkrankheiten im Juliusspital gemacht haben.

Meine Beobachtungen datiren aus dem Jahre 1888. Es wurden im Ganzen 13 Fälle ron Psoriasis mit Jodkali behandelt, deren Krankengeschichten im Folgenden mitgetheilt werden:

$$
\text { I. Gruppe. }
$$

1. S. Rosine, 17 Jahre alt, Dienstmädchen. Heredität nicht nachweisbar. Patientin war schon vom 11./IX. 1886 bis 10./VIII. 1887 wegen Psoriasis universalis in Spitalbehandlung und mit den verschiedensten inneren und äusseren Mitteln behandelt worden, bis sie schliesslich als temporär geheilt entlassen werden konnte. Schon 2 Monate nach der Entlassung traten an den Streckseiten der Extremitäten wieder PsoriasisFlecke auf, welche die Patientin zu abermaligem Eintritt in das Spital veranlasstea. Status 3./RV. 1888. Patientin gross, kräftig gebant, wohIgenährt.

Psoriasis guttata et numularis am Rumpfe und an den Extremitäten; Handteller, Fusssohlen und Gesicht frei.

Körpertemperatur normal, Puls 80.
1) La policlinique. 6. 1892.
$\left.{ }^{2}\right)$ Volkm. Vorträge. Nr. 93.
3) Arch. f. Dermat. 1892.
4) Archiv f. Dermat. u. Syphil. XXIV. 2. 1892 p. 341.
5) Brit. med. Journ. 28. Oct. 1893. 
Ueber die Behandl. der Psoriasis mit grossen Dosen von JK. 329

Ordination: Kal. jodat. 10॰0, Aq. destil. 200.0 4 Mal täglich 1 Esșlöffel voll.

19./IV. Die Haut der Extremitäten durch Abfall des grössten Theiles der Schuppen and Blasser- und Flacherwerden der Plaques glatter geworden. Dả Patientin über Magenbeschwerden klagt, muss Jodkali für einen Tag ausgesetzt werden. Die Temperatur ist in diesen Tagen normal geblieben, die Pulsfrequenz hie und da auf 10E-112 gestiegen. Mixtur.

20./IV. Von heute ab wieder Jodkali 4 Mal 3 Esslöffel voll obiger

25./TV. Von heute an 15*0 Jodkali: 200.0 Aqua destill. 10 Esslöfel täglich.

8./V. Patientin ist zu 20 Esslöffel der genannten Mixtur gestiegen, klagt wieder über Magenschmerzen, so dass abermals ausgesetzt werden muss.

Temperatur normal geblieben, Puls ebenfalls im Allgemeinen normal, nur an einzelnen Tagen hat sich eine Erhöhung auf 100-104 gezeigt, ohne Herzklopfen und ohne Kurzathmigkeit. Allgemeinbefinden war bis heute sebr gut, Körpergewicht hat zugenommen.

Die Psoriasisplaques sind bedeutend abgeblasst, Schuppung nur noch sehr gering, nur am rechten Oberarm sind einige frische Plaques aufgetreten.

Patientin hat im Ganzen 370 Gr. Jodkali genommen. Ausser einem wöchentlichen Reinigungsbad war eine weitere Behandlung nicht vorgenommen worden. Therapie von jetzt an Chrysarobin-Traumaticin.

14./V. Die Wirkung des Chrysarobins ist im Vergleich zur Wirkung bei früherem Spitalaufenthalt eine überraschend gute.

Ueber den weiteren Verlauf ist nichts mitzutheilen, da Jodkali später nicht mehr gegeben wurde.

2. Ziegler Margarethe 18 Jahre alt, aufgenommen am 14./III 1888. In der Familie eine gleiche Erkrankung nicht beobachtet. Vor einem halben Jahre wurden die ersten Krankheitserscheinungen an den Armen und im Gesicht beobachtet. Vor 3 Wochen breitete sich der zuerst geringfügige Ausschlag rasch über den ganzen Körper aus.

Status. 14./1II. 1888. Patientin von mittlerer Grösse, kräftig gebaut, wohlgenährt. Der ganze Körper mit Ausnahme der Hohlhand und der Fusssohlen mit Psoriasis plaques besetzt (Psoriasis numularis), am Rumpf and im Gesicht confluiren die Plaques stellenweise. Ordination 2*0 Jodkali pro die.

23./WI. Die Dosis Jodkali ist pro die auf $4^{\circ} 0$ gesteigert, obne dass irgend welche Störungen des Allgemeinbefindens aufgetreten wären, Temperatur and Puls sind normal geblieben, die Schuppung im Gesicht und am Hals hat bedeutend abgenommen, die Plaques sind am ganzen Körper blasser geworden.

28./II. Patientin hat in den letzten Tagen je $5 \cdot 0$ Jodkali pro die genommen. Störungen des Allgemeinbefindens haben sich nicht bemerkbar gemacht. Temperatur stets normal. Pulsfrequenz einige Male stark 
erhöht, einmal bis zu 128 obne Herzklopfen, ohne Störungen des Allgemeinbefindens, die Psoriasis bedeutend vermindert. Körpergewichtszunahme 1 Pfund. Patientin hat bisher 45 Gr. Jodkali genommen, die Entlassung erfolgte auf dringenden Wunsch der Patientin.

In diesen beiden Fällen hat die innerliche Darreichung von Jodkali einen entschieden günstigen Einfluss auf die Psoriasis ausgeübt, leider konnte in dem ersten Falle die Medication wegen Magenschmerzen, in dem zweiten Falle wegen vorzeitigem Austritt aus dem Spital nicht genügend lange fortgesetzt werden.

In Fall 1 zeigte sich nach Aussetzen des Jodkali eine im Gegensatze zur früheren Spitalbehandlung auffallend rasche und günstige Wirkung der Localbehandlung mit Chrysarobin, entsprechend den Beobachtungen Fabry's. Abgesehen von den Magenbeschwerden in Fall 1 haben die verhältnissmässig grossen Dosen Jodkali das Allgemeinbefinden nicht gestört, das Körpergewicht hat in beiden Fällen unter dieser Behandlung zugenommen. Die Körpertemperatur blieb stets normal, dagegen wies die Pulsfrequenz eine zum Theil recht beträchtliche Steigerung auf, einmal bis zu 128. Ich werde auf diesen lezteren Punkt später noch zurückkommen.

\section{Gruppe.}

3. Weinert August, 39 Jahre alt. In der Familie ist die gloiche Erkrankung noch nicht beobachtet worden. Vor 12 Jahren Beginn der Erkrankung. Patient war schon mehrfach in Spitalbehandlung, hier zum 1. Male vom 8./VI.-27./VIII, 1887, er wurde damals mit Chrysarobin und Pyrogallussäure behandelt und als gebessert entlassen. Anfangs Januar 1888 bemerkte Patient wieder Ausschlag an den Oberarmen, in den folgenden Wochen verbreitete sich die Erkrankung ziemlich rasch über den ganzen Körper.

Status 3./VI. 1888: Patient mittelgross, sehr kräftig gebaut, wohlgenährt. Psoriasis numularis et gyrata verbreitet über Extremitäten, Rumpf, behaarten Kopf, Gesicht frei. An den volae man. und an den Plantae pedum einzelne Plaques.

Ordination: Von einer Jodkalimixtur $10: 200$ nimmt Patient täglich 4 Esslöffel voll.

5. VI. Patient hat mässig starken Jodschnupfen. Körpertemperatur morgens auf $38^{\circ} 0$ gestiegen, Puls auf 120 . Jodkali ausgesetzt.

7./VI. Jodschnupfen beseitigt, Temperatur und Puls normal.

15./VI. Die Dosis Jodkali war auf 12.0 gesteigert worden, die Temperatur normal geblieben, die Pulsfrequenz einige Male bis auf 100 gestiegen. Die Schuppen fangen an alozufallen und die Psoriasisplaques 
blasser zu werden, neue sind nur wenige hinzugekommen. Aussetzen der Medication wegen Magenbeschwerden vom 15.-19.

27./VI. Die Dosis des Jodkali war in diesen Tagen von $12 \cdot 0$ auf 16.0 gesteigert worden, hatte nur an einem Tage geringe Magenbeschwerden ver ursacht, so dass nicht ausgesetzt werden musste, Temperatur normal geblieben, Pulsfrequenz mehrmals auf 108 gestiegen. Die Psoriasisplaques an der Innenfläche und am grössten Theile des Rumpfes fast vollständig zurückgegangen.

11./VII. Die tägliche Dosis des Jodkali hat jetzt die Höhe von 32.0 erreicht. Temperatur und Pulsfrequenz normal geblieben. Am behaarten Kopfe, an den Streckseiten der Extremitäten noch eine Anzahl von Plaques, die von jetzt an einer localen Behandlung mit Chrysarobin unterzogen werden (am Kopfe Praecipitatsalbe). Daneben bekommt Pat. Jodkali weiter.

31./VII. Tägliche Dosis Jodkali 34.0. Die Psoriasisplaques sämmtlich beseitigt, aber nicht mit Hilfe des Chrysarobius, sondern mit Hilfe von Anthrarobin, da ersteres schon am ersten Tage eine heftige Dermatitis hervorgerufen hatte.

9./VIII. Pat. als temporär geheilt entlassen. Das Allgemeinbefinden war diese ganze letzte Zeit über ein vortreftliches, das Körpergewicht hatte eine Zunahme von $4.5 \mathrm{Ko}$. erfahren (von 65.5 auf 69.0 ). Anthrarobin hatte einen raschen und vollständigen Heileffect bedingt. Gesammtdosis des genommenen Jodkali 872 Gr.

4. Schlereth Bruno, 42 Jahre alt. Im Alter von 18 Jahren zum ersten Male an Psoriasis erkrankt, zum letzten Male vor 2 Jahren, damals mit Chrysarobin behandelt. Heredität nicht nachweisbar.

Status 11./V. 1888: Patient gross, kräftig gebaut, ziemlich gut genährt. An der Streckseite der Extremitäten Psoriasis numularis, am Rumpf und im Gesicht vereinzelte Plaques von verschiedener Grösse.

Ordination: Jodkali 3.0 pro die.

13./V. Schon gestern Abend ist die Pulsfrequenz auf 108 angestiegen, die Temperatur auf $37 \%$. Heute ebenfalls erhöhte Temperatur und Pulsfrequenz.

14./V. Fieber, Kopfschmerzen, Acne jodica. Jodkali ausgesetzt.

18./V. Temperaturabfall. Die Jodacne im Gesicht sehr ausgebreitet. 19./V. Von Neuem Jodkali zu 4.5 pro die.

24./V. Mässiger Jodschnupfen. Temperatur normal geblieben, aber die Pulsfrequenz häufig über 100 erhöht. Jodkali 7.0 pro die. Die Schuppen im Gesicht und am Rumpf abgefallen, die Plaques ganz blass, an den Extremitäten noch wenig Wirkung vom Jodkali zu bemerken.

30./V. Die Tagesdosis Jodkalium beträgt 11.0. Gesicht und Rumpf ganz frei von Psoriasis, an den Extremitäten sind die Schuppen abgefallen und die Plaques blass geworden. Jodkali ausgesetzt.

11./VI. Die letzten Reste der Psoriasis gingen ganz rasch weg auf Chrysarobin-Traumaticinanwendung. Obgleich in den Ietzten Wochen das Allgemeinbefinden ganz gut, Körpertemperatur normal geblieben war, ist 


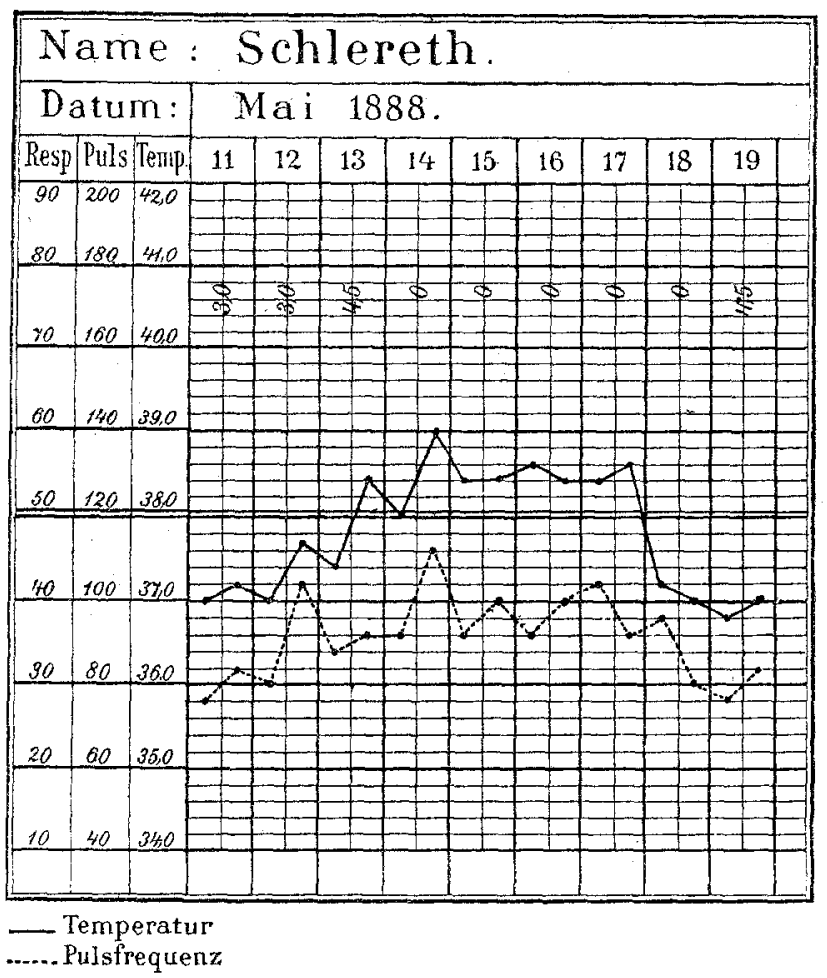

dennoch das Körpergewicht von $58 \cdot 5$ Kilo auf 55.0 Kilo heruntergegangen. Gesammtdosis des Jodkaliums $220 \mathrm{Gr}$.

5. Kuhn Pauline, 28 Jahre alt, Heredität nicht nachweisbar. Erstes Auftreten der Hautkrankheit im 18. Lebensjahre, seit dieser Zeit zahlreiche Rückfälle, mehrfache Spitalbehandlung.

Status 11./XI. 1888: Patientin kräftig gebaut, wohlgenährt, $P_{\text {soriasis }}$ guttata an den Streckseiten der Extremitäten, Psoriasis numularis in der Glutäalgegend, der übrige Rumpf und der Kopf frei.

Ordination: Kal. jodat. $3 \cdot 0$ pro die.

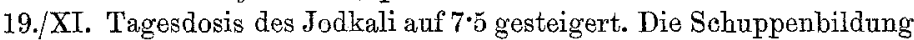
entschieden geringer, die Plaques wesentlich blasser geworden, neue Nachschube nicht beobachtet. Jodkali wurde bisher sehr gut vertragen, an einzelnen Tagen nur geringer Jodschnupfen und mässige Erhöhung der Pulsfrequenz bis auf 100 beobachtet, ohne Hexzklopfen oder anderweitige Störungen des Allgemeinbefindens.

8. XII. In der letzten Zeit auch Jodkali weniger gut vertragen, der Schnupfen machte sich in unangenehmerer Weise bemerkbar, dazu kamen auch öfters heftige Kopfschmerzen, so dass das Mittel einige Male ausgesetzt werden musste. Die Pulsfrequenz stieg manchmal bis zu 116 an, 
Ueber die Behandl. der Psoriasis mit grossen Dosen von JK. 333

die Körpertemperatur bis auf 37·. Die Besserung der Psoriasis hat in der letzten Zeit keine wesentlichen Fortschritte gemacht. Jodkali wird nun ganz ausgesetzt und die Reste der Psoriasis sollen mit Chrysarobin-Traumaticin beseitigt werden.

7./I. 1889. Patientin als temporär geheilt entlassen. ChrysarobinWirkung war eine sehr rasche und vollständige. Körpergewicht um 1 Kilo gestiegen (von 62.5 auf 63.5). Gesammtdosis des verabreichten Jodkalium 270 Gr.

6. Beck Georg, 16 Jahre alt, Heredität nicht nachweisbar. Patient will die Hautkrankheit schon in frühem Kindesalter bekommen haben.

Status 21./II. 1889: Patient kräftig gebaut, wohlgenährt. Psoriasis punctata et numularis der Extremitäten, des Rumpfes und des behaarten Kopfes; Gesicht und Hals frei. pro die.

Ordination: Kali jodat 1.5

24./II. Kali jod. 3.0 pro die.

26./II. Gestern Abend Steigerung der Pulsfrequenz auf 108, Temperatur war normal. Heute Morgen 96 Puls, Temperatur $366^{\circ}$. Pat. klagt über Kopfschmerzen. Abends plotzliche Temperatursteigerung auf 39.4 , Puls 100. Patient hatte seine Tagesdosis schon genommen. Keine Störung des Allgemeinbefindens.

27./I. Heute Temperaturabfall, Allgemeinbefinden besser, da Patient seit gestern kein Jodkali mehr genommen hat.

28./II. Da wieder ganz normale Verhältnisse bestehen, so nimmt Patient von Neuem Jodkali $3 \cdot 0$ pro die.

19./IIr. Patient hat dieses Mittel seither regelmässig genommen, ohne dass eine erneute Störung des Befindens eingetreten wäre, nur die Pulsfrequenz hat an einzelnen Tagen eine Steige-
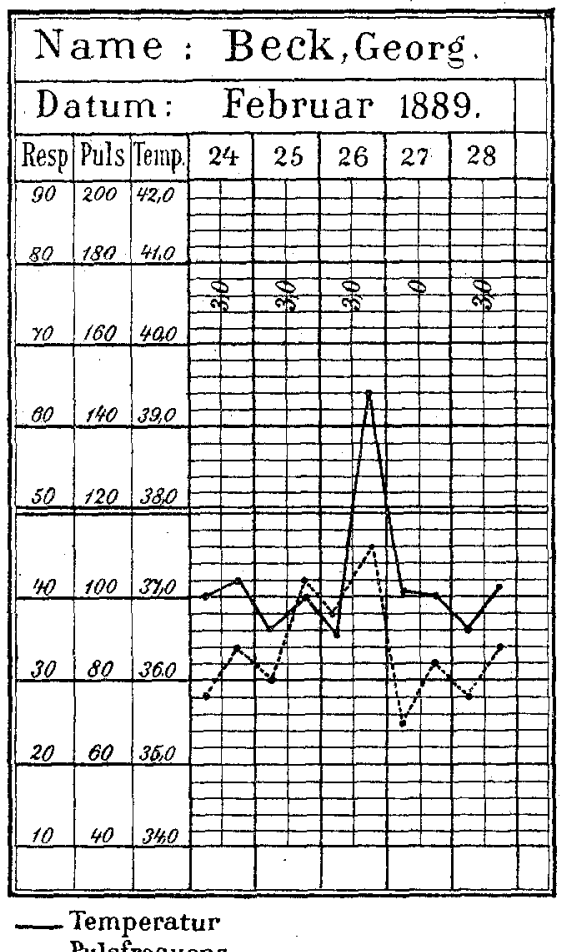

rung bis zu 116 erfahren. Die Psoriasisefflorescenzen ganz abgeblasst, Schuppenbildung nur noch sehr gering. Um den Rest der Psoriasis rascher zu beseitigen, wird Chrysarobin-Traumaticin applicirt, Jodk. weitergegeben.

23./III. Patient vollkommen geheilt. Eine Störung des Allgemeinbefindens ausgeblieben. Patient hat im Ganzen $136 \mathrm{Gr}$. Jodkalium genommen. Körpergowicht ist um 4 Kilo gestiegen (von 40 auf 44). 
7. Höppel Joseph, 28 Jahre alt, Beginn der Erkrankung vor $1 / z$ Jahre. Heredität nicht nachweisbar.

Status 12./IX. 1888: Patient schwächlich gebaut. Psoriasis numularis et figurata an den Extremitäten and am Rumpfe, am Kopfe nur die Stirne befallen.

Ordination: Kali jodat 3.0 pro die.

10./X. 1888. Die tägliche Dosis des Jodkali auf $16 \cdot 0$ gesteigert, Allgemeinbefinden, abgesehen von leichtem Jodschnupfen, nicht gestört. Temperatur bis heute normal geblieben, heute Abend (Patient nimmt heute zum 1. Male 16.0) eine mässige Temperatursteigerung auf $38^{\circ} 0$, Pulsfrequenz hat sich in den letzten 6 Tagen ständig zwischen 108 und 124 bewegt, heute Abend aber 132 erreicht. Allgemeinbefinden vollkommen normal.

11./X. Jodkali ausgesetzt. Temperatur normal, Puls auf 120 heruntergegangen. Die Psoriasisefflorescenzen wesentlich abgeblasst, die Schuppung geringer. Die Reste der Psoriasis sollen mit Chrysarobin behandelt werden.

15. X. Psoriasis fast ganz geheilt, Patient wird auf seinen dringenden Wunsch entlassen. Allgemeinbefinden in den letzten Tagen sehr gut. Patient hat im Ganzen 210 Gr. Jodkali genommen. Körpergewicht um 4 Kilo gestiegen (von 45 auf 49 ).

8. Riehwar Michael, 21 Jabre. Erstmalige Erkrankung vor 1 Jahre. Heredität nicht nachweisbar.

Status 27./XII. 1887. Patient ziemlich kräftig gebaut, gut genäbrt. Psoriasis punctata et guttata über den ganzen Körper ausgebreitet.

Vom 10./. 1888 an nimmt Patient 3.0 Kali jod. pro die.

19./I. In der ersten Zeit musste einige Male wegen beftigen Jodschnupfens die Medication ausgesetzt werden. Temperatur und Pulsfrequenz bisher normal. Heute zum 1. Male Temperatursteigerung auf 38.6 und Erhöhung der Pulsfrequenz auf 112. Jodkali ansgesetzt ohne Störung des Allgemeinbefindens.

28./II. Die tägliche Dosis Jodkali ist allmälig auf $27 \mathrm{Gr}$. gesteigert worden. Im Allgemeinen hat Patient dieses Mittel gut vertragen, jedoch bei jeder Steigerung der Tagesdosis eine Frhöhung der Körpertemperatur auf 37.9-38.7 und der Pulsfrequenz auf 100-120 erfahren, ohne dass sich irgend welche Störungen des Allgemeinbefindens bemerkbar gemacht hatten. Da diese Erscheinungen ganz regelmässig eintraten, und am 2. Tage ohne Aussetzen der Medication wieder zurückgingen, so wurde eine Unterbrechung der Cur nicht für nöthig gehalten, zumal die Wirkung des Jodkali auf die Psoriasis unverkennbar war. Die Fifflorescenzen sind im Gesich t und am Rumpfe vollkommen zurückgegangen, so dass hier die Haut ganz glatt erscheint. An den Extremitäten findet sich noch eine Anzahl schuppender Plaques, die zu rascherer Beseitigung mit Chrysarobin-Traumaticin behandelt werden sollen.

11./III. 1888. Chrysarobin hat sehr rasch und gründlich gewirkt. Patient heute geheilt entlassen. Gesammtdosis des verabfolgten Jodkali: 414 Gr. Körpergewicht war bis zum 27./I. von 51 auf 52.5 Kilo gestiegen 
Ueber die Behandl. der Psoriasis mit grossen Dosen von JK. 335

und ist zuletzt bis auf 51.5 zurückgegangen, so dass Patient immerhin noch mit einer $Z$ unahme von 0.5 Kilo das Spital verlässt.

9. Weber Karl, 38 Jabre. Heredität nicht nachweisbar. Erste Erkrankung im 20. Lebensjahre. Patient schon mehrfach in Spitalbehandlung gewesen. Vor 16 Wochen Recidiv.

Status: 26./IX. 1888. An den Extremitäten und am Rumpf Psoriasis guttata et numularis in geringer Ausdehnung, an der Stirne Psoriasis gyrata.

Ordination: Kali jodat. $2 \cdot 0$ pro die.

11./X. Die tägliche Dosis bereits gesteigert auf 14*0. Während bisher Jodkali sehr gut vertragen wurde, weder Temperatursteigerung

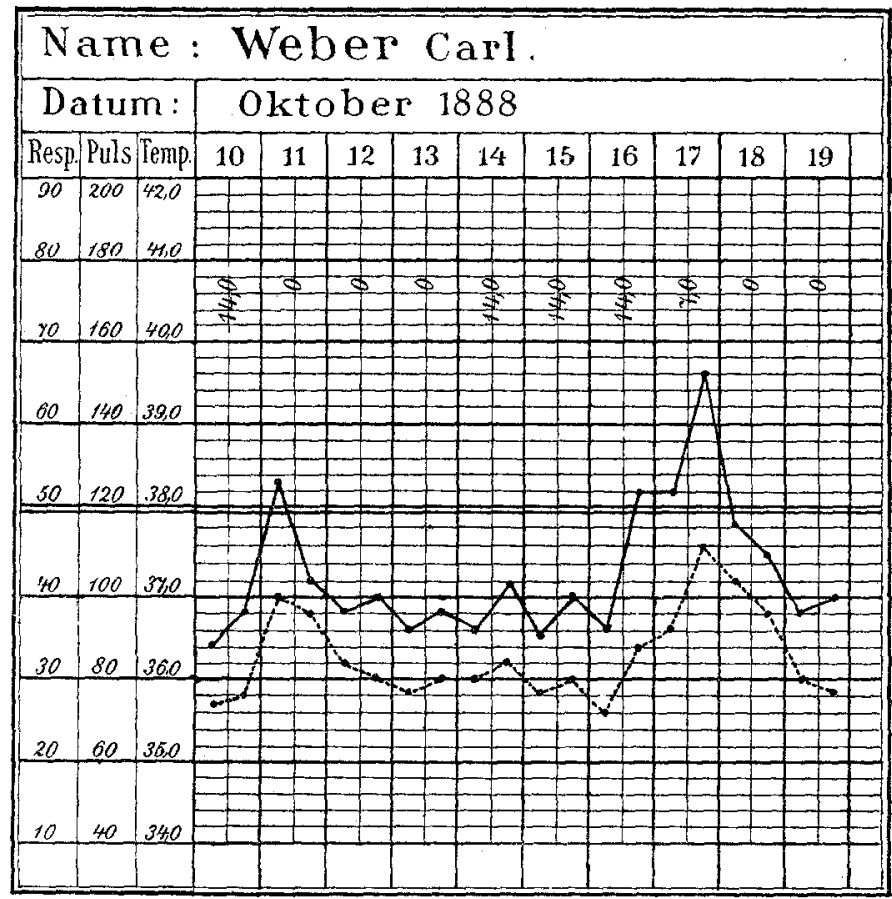

Temperatur

...... Pulsfrequenz

noch Erhöhung der Pulsfrequenz sich zeigte, klagt Pat. heute über allgemeines Unbehagen, Kopfschmerzen. Temper. 38*3, Puls 100. Jodkali ausgesetzt. 12./X. Temp. und Puls wieder normal, Allgemeinbefinden gut.

14./X. Von heute ab wieder $14^{\circ} 0 \mathrm{Kal}$. jodat. 17./X. Gestern Abend und heute Morgen Fieber. Patient bekommt heute noch die halbe Dosis Jodkali. Trotz der hohen Abendtemperatur keine Störung des Allgemeinbefindens, ausser dem Gefühl grosser Müdigkeit. 18./X. Wohlbefinden, normale Temperatur, Pulsfrequenz noch etwas hoch. Vom 21./X. an wieder Jodkali $12 \cdot 0$ pro die. 
2./XI. Mit der täglichen Jodkali-Dosis war bis zu 16.0 gestiegen worden, ohne dass mehr störende Nebenwirkungen zur Beobachtung gelangten. Pat. hat im Ganzen 268 Gr. Jodkali genommen. Körpergewicht trotz der zweimaligen Fieberanfälle um 2 Kilo gestiegen (von 60 auf 62). Die Psoriasisplaques am Rumpfe und an den Extremitäten vollkommen beseitigt, nur an der Stirne noch etwas Röthung und Schuppung.

Therapie: Antbrarobin.

15. XI. Patient geheilt entlassen.

\section{Gruppe.}

10. Deubert Katharine, 26 Jahre. Die Mutter der Patientin soll an derselben Hautkrankheit gelitten haben. Im 20. Lebensjahre erstmaliger Ausbruch der Erkrankung, seither war Pat. schon mehrmals in Spitalbehandlung. Vor 3 Wochen Auftreten neuer Erscheinungen.

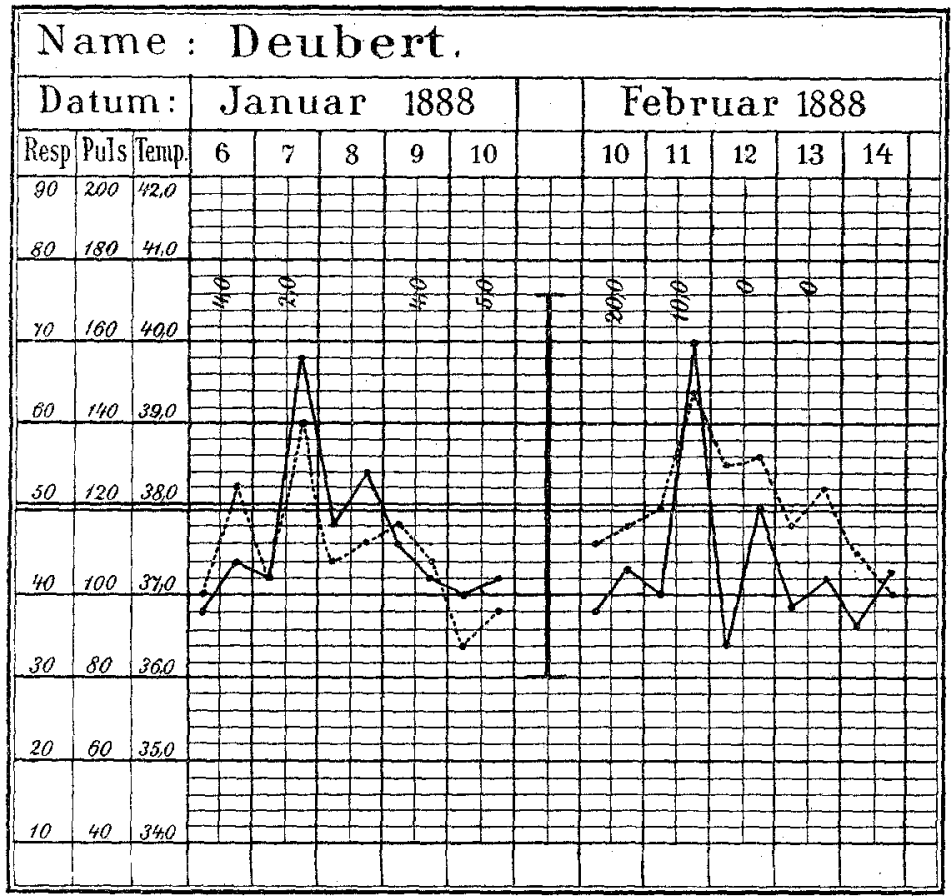

- Temperatur

...... Pulsfrequenz

Status 27./XII. 1887. Am Rumpf und an den Extremitäten Psoriasis punctata. Gesicht und Kopf frei.

Ordination: Kal. jodat. 3*0 pro die. 
Ueber die Behandl. der Psoriasis mit grossen Dosen von JK. 337

7./. Die tägliche Dosis des Jodkali gesteigert auf $4^{\circ} 0$. Abgesehen von einem leichten Jodschnupfen traten bisher unangenehme Störungen nicht auf, auffällig war nur, dass gestern Abend die Pulsfrequenz über 126 gestiegen war. Heate Abend plötzliche Temperatursteigerung und bedeutendere Erhöhung der Pulsfrequenz. Patientin klagt über allgemeine Müdigkeit und starke Schlingbeschwerden, als deren Ursache eine erythematöse Röthung der Gaumen- und Rachenschleimhaut mit ödematöser Anschwellung der Uvula anzusehen ist. Abends Jodkali ausgesetzt.

8./I. Oedem der Uvula zurückgegangen, Erythem etwas geringer. Schlingbeschwerden vermindert. Allgemeinbefinden besser.

9./I. Schleimbaut der Mund- und Rachenhöhle wieder nahezu normal. Jodkali fortgesetzt.

11./II. 1888. Die tägliche Dosis des Jodkali auf $20^{\circ} 0$ gesteigert. Allgemeinbefinden seither nicht mehr gestört. Auffällig ist, dass seither die Pulsfrequenz bei normalem Verhalten der Temperatur eine häufige Steigerung auf 100-128 erfuhr. Heute Abend Temperatursteigerung und bedeutende Erhöhung der Pulsfrequenz ohne irgend welche locale Störungen, nur Gefühl von grosser Müdigkeit, Jodkali ausgesetzt.

12./II. Allgemeinbefinden ganz gut.

14./II. Die Schuppen überall abgefallen, die Plaques vollkommen verschwunden. Patientin gebeilt entlassen.

Patientin hat im Ganzen 422 Gr. Jodkali genommen. Das Körpergewicht war bis Mitte Februar um 1.5 Kilo gestiegen, ist aber in den letzten Tagen des Spitalaufenthaltes auf das Anfangsgewicht zurückgegangen.

11. Hofling Margarethe, 23 Jahre. Hereditär nicht nachweisbar. Die ersten Krankheitserscheinungen traten vor $1 / 2$ Jahre an den Knien und Ellenbogen auf.

Status 9.II. 1888. Pat. kräftig gebaut, wohlgenährt. Psoriasis guttata et numularis. Sitz: Rumpf und Extremitäten. Kopf und Gesicht frei.

Ordination: Kal. jodat. $3 \cdot 0$ pro die.

12./II. Steigerung der täglichen Dosis Jodkali auf 19・0. Bisher war das Allgemeinbefinden ein gutes, hie und da machten sich Magenbeschwerden bemerkbar, welche rasch wieder vorübergingen. Einige Male mässige Erhöhung der Pulsfrequenz bei durchweg normaler Temperatur.

21./II. In den letzten Tagen ganz auffällige Steigerung der Pulsfrequenz bei ganz normaler Temperatur. Allgemeinbefinden dabei nicht gestört.

10./III. In den letzten Tagen mässige Erhöhung der Temperatur bis auf 38.4 und der Pulsfrequenz auf 100-120 ohne Störungen des Allgemeinbefindens. Die Patientin befindet sich ganz wohl, die Schuppen und somit auch die Plaques vollkommen beseitigt, so dass Pat. als geheilt bezeichnet werden kann. In Summa hat Pat. 850 Gr. Jodkali genommen. Körpergewicht hat um 2 Kilo zugenommen $(57 \cdot 7$ auf $59 \cdot 7)$.

Arehiv f. Dermatol. u. Syphil. Band XXVII. 


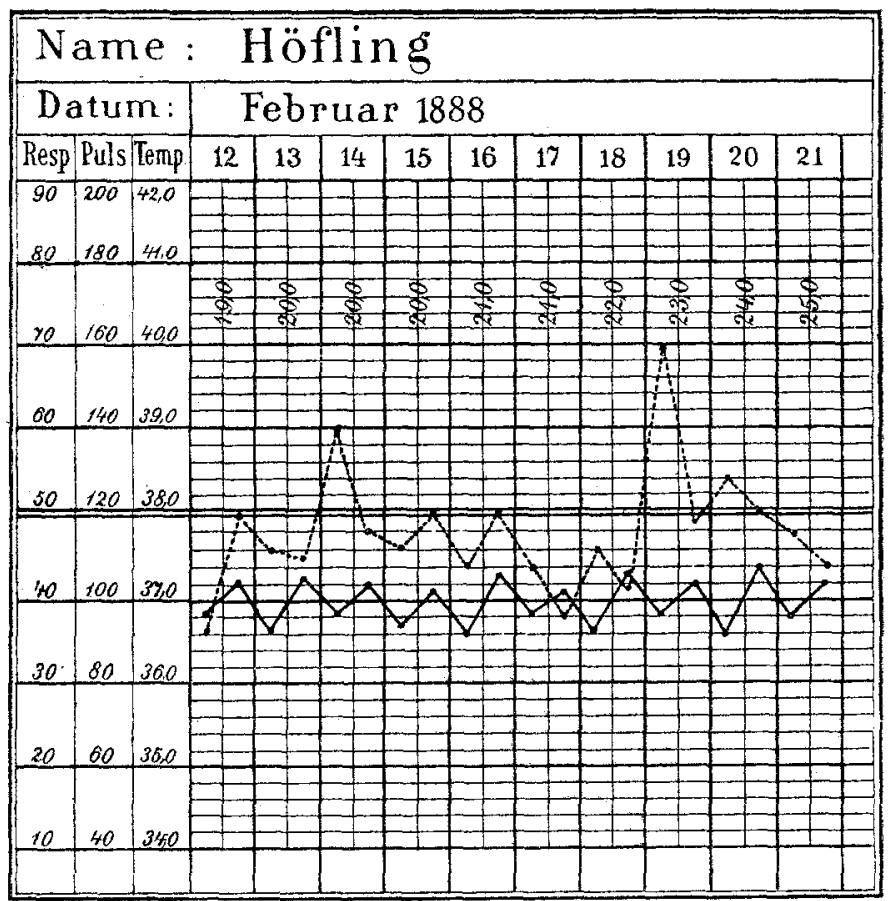

Temperatur

.......Pulsfrequenz

12. Haas Eva, 17 Jahre alt. Heredität nicht nachweisbar, Erste Krankbeitserscheinungen vor 4 Jahren, Pat. ist schon mehrfach behandelt worden.

Status 16./XI. 1887. Pat. ziemlich gut entwickelt, wohlgenährt. Psoriasis numularis et guttata. Sitz: Rumpf und Extremitäten. Gesicht und Hals frei.

Ordination: Kali jodat. 1.0 pro die.

20./XI. Abends. Unter Temperatursteigerung und erheblicher Erhöhung der Pulsfrequenz ist ein urticariaähnliches Exanthem aufgetreten, das nahezu über den ganzen Körper verbreitet ist. Jodkali nicht ausgesetzt.

24./XI. Das Exanthem zurückgegangen. Temperatur normal, Pulsfrequenz noch erhöht.

19./. 1888. Derartige Attaquen sind noch 5 Mal ganz in derselben Weise wie zum ersten Male aufgetreten, dabei wurde Jodkali nie ausgesetzt, sondern in langsam ansteigender Dosis fortgegeben. Der ganze Körper frei von Psoriasisplaques. Pat. als geheilt zu betrachten.

Im Ganzen wurden $344 \mathrm{Gr}$. Jodkali genommen. Das Körpergewicht war anfangs von $36^{\circ} 0$ auf $38^{\circ} 0$ Kilo gestiegen, ist aber später auf $36^{\circ} 5$ Kilo zurückgegangen. 
Ueber die Behandl. der Psoriasis mit grossen Dosen von JK. 339

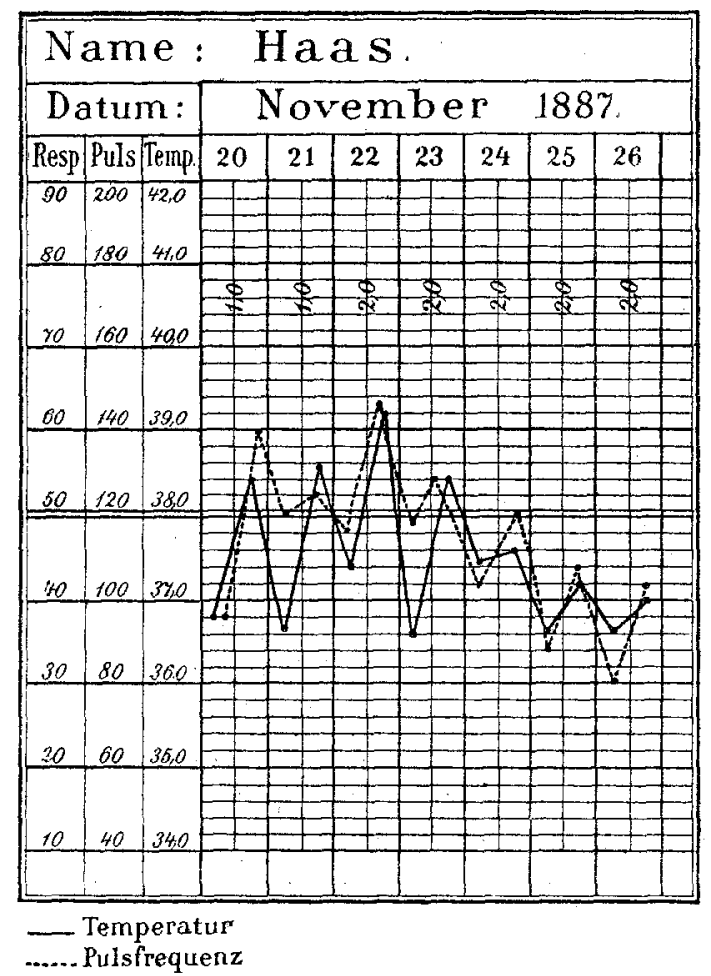

13. Melber Michael, 48 Jahre alt. Der Vater des Patienten litt an der gleichen Hantkrankheit. Erstmalige Erkrankung vor 14 Jahren. Die jetzige Erkrankung begann vor 8 Tagen unter Störungen des Allgemeinbefindens; Müdigkeit, Frösteln.

Status 30./I. 1889. Pat. gross, kräftig gebaut. Psoriasis numularis et gyrata. Sitz: Rumpf und Extremitäten, Gesicht und Hals frei.

Ordination: Kal, jodat. 1.5 pro die.

5./III. 1889. Im Allgemeinen wurde Jodkali gut vertragen. Einmal erhob sich die Körpertemperatur auf 38.2 , um am folgenden Tage wieder normales Verhalten zu zeigen. Sebr oft wurde eine Steigerung der Pulsfrequenz auf 100-128 beobachtet. Die Psoriasis vollkommen geheilt.

In Summa hat Pat. 223 Gr. Jodkali genommen. Körpergewicht um 4 Kilo gestiegen.

Die vorliegenden 13 Krankengeschichten habe ich deshalb in drei Gruppen getheilt, weil einmal die Wirkung des Jodkaliums auf die Psoriasis und andererseits eine Reihe von Nebenwirkungen des Jodkalium durch solche Eintheilung in iibersichtlicherer Weise zu besprechen ist. Was die Form der Psoriasis. 
anlangt, so hatte dieselbe in ihrer Localisation, mit Ausnahme eines Falles, nichts, was sie von den gewöhnlich zu beobachtenden Formen der Psoriasis vulgaris unterschied.

Bei einem Falle (Nr. 13) handelte es sich um einen acuten Ausbruch der Erkrankung unter Störungen des Allgemeinbefindens, ein Fall (Nr. 2) kann als Psoriasis universalis bezeichnet werden, in welchem nur Handfläche und Fusssohle frei blieben, ein Fall (Nr. 10) wird als Psoriasis punctata aufgefïhrt, ein Fall (Nr. 8) als Psoriasis punctata et guttata, ein Fall als Psoriasis punctata et numularis (Fall Nr. 6), ein Fall (Nr. 4) als Psoriaris numularis, 3 Fälle als Psoriasis guttata et numularis (Nr. 5, 9 und 11), 3 Fälle als Psoriasis numularis et gyrata (Nr. 1, 7 und 12). Der Fall 3 zeichnet sich dadurch aus, dass bei ihm nicht nur der ganze Körper in Form der Psoriasis numularis et gyrata befallen war, sondern dass sich auch an den Handflächen und an den Fusssohlen einige typische Plaques fanden. Es ist das bekanntermassen eine bei der Psoriasis vulgaris sehr selten zu beobachtende Localisation. (Nobl.) ${ }^{1}$ )

Heredität war nur in 2 Fällen (Nr. 10 und Nr. 13) nachweisbar, in einem Falle hatte die Mutter, in dem zweiten Falle der Vater an der gleichen Hautkrankheit gelitten. (In dem Falle 10 begann die Erkrankung im 20., bei Nr. 13 im 30. Lebensjahre.) Die Mehrzahl der Fälle hatte schon eine grössere Anzahl von Attaquen durchgemacht und sich wegen solcher theils in Privat-, theils in Spitalbehandlung befunden, in einem Falle kam das erste Recidiv hier zur Behandlung, und 5 Fälle wurden behufs erstmaliger Behandlung in die Klinik aufgenommen.

Die ersten Krankheitserscheinungen sollen bei Nr. 6 schon in den ersten Lebensjahren, bei Nr. 12 im 13. Lebensjahre beobachtet worden sein, bei den 11 anderen Fällen traten sie erstnach der Pubertät auf und zwar in 6 Fällen zwischen 15 . und 20. Lebensjahr, in 5 Fällen zwischen 20. und 30. Lebensjahr.

Von diesen 13 Fällen betrafen 7 männliche Individuen, die alle wohlgenährt und kräftig gebaut waren, und 6 weibliche Individuen, von denen nur eines schwächlich gebaut war und schlechten Ernährungszustand aufwies, während die übrigen 5

1) Wien. dermat. Gesellsch. 22. Nov. 1893. 
Ueber die Behandl. der Psoriasis mit grossen Dosen von JK. 341

ebenso wie die Männer als kräftig und wohlgenährt zu bezeichnen waren.

Die Eintheilung der ersten 9 Fälle in II Gruppen bezieht sich weniger auf die Wirkung des Jodkalium auf die Psoriasis, als auf gewisse Nebenwirkungen des genannten Mittels, welche nachher zur Besprechung kommen sollen. In Bezug auf den ersteren Punkt verhalten sich Gruppe I und II gleich, insofern bei diesen Fällen eine vollständige Heilung der Psoriasis durch Jodkali nicht erreicht worden war. Eine vollkommene Heilung der Psoriasis durch Jodkalium konnte nur in den 4 Fällen der II. Gruppe erzielt werden, welche in Bezug auf die Nebenwirkungen zusammen mit den Fällen der II. Gruppe besprochen werden sollen.

Unter den 9 Fällen der I. und II. Gruppe konnte in Nr. 2 nur eine Besserung der Psoriasis verzeichnet werden, die Patientin verliess, nachdem sie eine Gesammtdosis von 45 Gr. Jodkali genommen hatte, frühzeitig das Spital. Im ersten Falle musste wegen Magenschmerzen mehrfach das Jodkalium ausgesetzt und schliesslich, nachdem Patientin die Gesammtdosis von $370 \mathrm{Gr}$. genommen hatte, das Mittel vollkommen von der Tagesordnung abgesetzt werden, weil die Patientin sich andauernd über die genannten Beschwerden beklagte. Es war das gerade in diesem Falle um so beklagenswerther, weil es sich nach den Erfahrungen von einem früheren Spitalaufenthalt her um eine sehr schwere Form der Psoriasis handelte und weil gerade hier Jodkali auf die Hauterkrankung einen entschieden günstigen Einfluss geäussert hatte, so dass wir hätten erwarten können, bei consequenter Fortsetzung der Jodkalibehandlung eine vollkommene Heilung zu erzielen.

Wie bei diesem Falle, so zeigte sich auch bei den übrigen 7 Fällen der I. resp. II. Gruppe nach dem Aussetzen des Jodkali eine auffällig rasche Wirkung der localen Application von Chrysarobin resp. Anthrarobin und zwar in einer solchen Weise, dass man zu der Annahme gelangen musste, dass, wenn auch Jodkalium in einer Anzahl von Fällen nicht im Stande ist, Psoriasis zu heilen, dieses Mittel doch die Krankheitserscheinungen in einer solchen Weise verändert, dass die locale Behandlung zu einer sehr raschen Heilung führen kann. Ob zur 
definitiven Heilung sich nur die genannten Mittel (Chrysarobin und event. Anthrarobin) oder auch andere, wie Theer eignen, dariiber kann ich mir ein Urtheil nicht erlauben, weil wir nur vom Chrysarobin und Anthrarobin Gebrauch machten. Sehe ich ab von dem Fall 2, in welchem nur 45 Gr. Jodkali zur Verwendung kamen, so wurden in den 8 Fällen der I. und II. Gruppe Gesammtdosen Jodkalium von 136--872 Gr. gegeben.

Unter den 4 Fällen von vollkommener Heilung der Psoriasis waren 3 weiblichen, 1 männlichen Geschlechtes. In dem letzteren Falle handelte es sich um einen unter Störungen des Allgemeinbefindens aufgetretenen acuten Nachschub, der innerhalb 5 Wochen durch Jodkalium (223 Gr.) geheilt wurde.

Von den weiblichen Individuen war eine (Nr. 11) noch nicht behandelt worden, bei den anderen lagen chronische Formen vor, welche schon mehrfachen Heilungsversuchen getrotzt hatten. Die Heilung war auch bei diesen 3 eine vollkommene ausschliesslich der Wirkung des Jodkali zuzuschreibende. Die Behandlungsdauer betrug im Durchschnitt 7 Wochen, also ebenso viel, als Haslund in seinen durch Jodkali geheilten Fällen gefunden hat.

Die verbrauchten Dosen Jodkalium beziffern sich auf $344-850 \mathrm{Gr}$.

Leider ist in meinen Fällen nicht genügend darauf geachtet worden, zu welchem Zeitpunkte die retrograde Metamorphose der einzelnen Efflorescenzen sich zu zeigen begann, im Allgemeinen glaube ich mit $\mathrm{Ha}$ s lu nd constatiren zu können, dass 4-5 Wochen der Zeitpunkt zu sein scheint, wo der Rückgang gewöhnlich anfängt. Auch darüber vermag ich nichts Sicheres anzugeben, an welchen Körperstellen sich dieser Rückgang zuerst bemerkbar macht, nach Greve und Haslund sollen die Efflorescenzen zuerst am Kopf und am Hals, dann am Rumpf ron oben nach unten und zuletzt an den Extremitäten rerschwinden. Das Eine habe ich mit grosser Regelmässigkeit beobachtet, dass die Efflorescenzen an den Extremitäten am hartnäckigsten sind.

Bei so grossen, für eine Reihe von Wochen in gleichmässiger Steigerung fortgesetzten Dosen Jodkali wird man sich 
die Frage vorlegen müssen, welche unangenehmen Nebenerscheinungen bei solchen, man kann wohl sagen, heroischen Curen zu beobachten sind. In Uebereinstimmung mit den Beobachtungen der Eingangs genannten Autoren zeigen auch meine Beobachtungen das auffällige Resultat, dass im Allgemeinen die hohen Tagesdosen Jodkali erstaunlich gut vertragen werden.

Derartige Beobachtungen sind gewiss nicht neu, verdienen aber immer wieder besonders hervorgehoben zu werden. Die Syphilidologen haben ja Gelegenheit, Aehnliches zu sehen. So. führt $\mathrm{Kop} \mathrm{p}^{1}$ ) an, dass manche Individuen $20-30 \mathrm{Gr}$. Jodkalium im Tage ohne irgend welche belästigende Symptome zu sich nehmen konnten. Garnett ${ }^{2}$ ) hat bei einem Falle von syphilitischer Spinalerkrankung 30-60 Gr. Jodkali pro die und im. Ganzen $12 \mathrm{Kg}$. Jodkali gegeben. Auch Segnin ${ }^{3}$ ) ist ein Anhänger von grossen Dosen Jodkalium (10-40 Gr. pro die) und scheut auch bei Kindern grosse Dosen nicht. Ueber einen Fall. von Jodtoleranz berichtet Ne u mann. ${ }^{7}$ ) Ein Füselier hatte eine ihm in der Dosis von 3 Mal täglich 1 Esslöffel zu nehmende Lösung von 20 Gr. Jodkali auf 400 Gr. Wasser in nicht ganz 24 Stunden getrunken, ohne dass sich irgend welche unangenehme Erscheinungen gezeigt hätten. Wolff ${ }^{5}$ ) hatte Gelegenheit bei 4 Fällen von schwerer tertiärer Syphilis Toleranz gegen grosse Dosen Jodkalium zu beobachten. In einem Falle wurden schliesslich 50 Gr. pro die drei Monate lang (im Ganzen 5 Kilo Jodkali) gegeben, ohne dass unangenehme Nebenwirkungen auftraten, das Körpergewicht war schliesslich um 28 Pfd. gestiegen., Im 2. Falle kamen 30 Gr. pro die 2 Monate lang zur Verwen-; dung, das Körpergewicht hatte am Ende der Cur um 21 Pfd. zugenommen. Im 3. Falle wurden $16 \mathrm{Gr}$.pro die, im 4 . Falle $32 \mathrm{Gr}$. Jodkali pro die vertragen. Die Erscheinungen des Jodismus: glaubt W. dadurch vermieden zu haben, dass er Jodkalium nicht. in Wasser, sondern in Amylum verabreichte. Graydon ${ }^{6}$ ) war

) Leirbuch der vener. Erkrankungen p. 517.

$\left.{ }^{2}\right)$ Ref. Deutsche Mediz. Zeitung. 1888.

$\left.{ }^{3}\right)$ Arch. d. méd. XII. p. 114:

4) Deutsche Med. Zeitung. 1889. 39 p. 458.

5) 62. Naturforseher-Vers. Heidelberg 1889

$\left.{ }^{6}\right)$ Med. and surg. report. Vol. 61. Nr. 11 . 
bei einem Pat. mit Chorioiditis syphilitica schliesslich auf $30^{\circ} 0$ Jodkali pro die gestiegen, ohne dass sich ausser Acne faciei unangenehme Nebenerscheinungen eingestellt hatten.

Montgommery ${ }^{1}$ ) ist in einem Falle, wo die Diagnose Syphilis zweifelhaft war, bis zu 120 Gramm Jodkali pro die gestiegen.

Zur Erklärung dieses eigenthümlichenVerhaltens des menschlichen Organismus gegenüber einem so differenten Mittel wie dem Jod muss an verschiedene Möglichkeiten gedacht werden. Einmal liesse sich denken, dass grosse Dosen Jodkalium diuretisch wirken und deshalb rascher ausgeschieden werden als kleine Dosen, die nicht diuretisch wirken. Für diese Annahme würde auch die schon mehrfach gemachte Beobachtung sprechen, dass gerade bei kleinen Dosen häufiger die Erscheinungen des Jodismus auftreten als bei den grossen Dosen, wie sie z. B. auf einmal in dem Falle von $\mathrm{Neumann}{ }^{2}$ ) genommen wurden. Bringier ${ }^{3}$ ) hat schon nach 0.32 Gr. Jodkali (3mal täglich genommen) acuten Jodismus auftreten sehen und B r u n thaven ${ }^{4}$ ) konnte die gleiche Beobachtung machen. De la Barcerie ${ }^{5}$ ) sah bei einem Falle von Angina pectoris schon nach $0.5 \mathrm{Gr}$. Jodkali die Erscheinungen des acuten Jodismus. In einem von $\mathrm{Russel}^{6}$ ) mitgetheilten Fall trat nach 3tägigem Gebrauch von Jodkali in kleinster Dosis acuter Jodismus auf, der unter Inauction und leichter Pneumonie zum Exitus führte. Möglicherweise handelte es sich hier um das Eintreten von Lungenödem, dessen Entstehung Zeissl ${ }^{7}$ ) experimentell an Hunden nachgewiesen hat in Uebereinstimmung mit den älteren Versuchen von $\mathrm{B} \ddot{\mathrm{hm}}$ und $\mathrm{Berg}{ }^{8}$ ). Diesen Thierexperimenten gegenüber erscheint die klinische Beobachtung sehr auffallend, dass Jodkali zur richtigen Zeit angewandt zur Abortiveur bei Pneumonie

1) St. Louis Med. and surg. Journ. 1893.

2) 1 . c.

3) Med. News. Jnli 1892.

4) Med. News. Sept. 1892.

5) Revue gén. đ. clin. et de thérap. 27. 1892.

$\left.{ }^{6}\right)$ New York med. record. Aug. 1893.

7) Intern. klin. Rundschau. 4. 1894.

8) Arch. f. exper. Path. u. Pharm. V. 4. u, 5, 1876. 
dienen könne. Velten ${ }^{1}$ ) gibt an, dass in jenen Fällen von Pneumonie, in welchen 12-22 Stunden nach dem Schüttelfrost mit dem Einnehmen des Mittels begonnen wurde, die Temperatur bis zur Norm sank, am Mittag oder Abend des zweiten Tages sich wieder erhob, aber gewöhnlich nur wenig über $38.0^{\circ}$ und dass eine zweite Gabe erforderlich war, um das Fieber dauernd zu beseitigen. Später als 24 Stunden nach dem Beginn dex Erkrankung werde rom Jod kein Exfolg mehr gesehen. Unangenehme Folgen der Einführung von Jodkali (6 Gr. in wenig Stunden) sah Velten nur in einem Falle (Oedem der Augenlider, der Oberlippe und des rechten Gaumenbogens, Halsschmerzen, Heiserkeit).

Die von Bresgen ${ }^{2}$ ) beobachteten Fälle von acutem Jodismus traten nach verhältnissmässig kleinen Dosen von Jodkali auf, so dass er an eine Verunreinigung des Präparates gedacht hatte. Nach der Untersuchung desselben von Bin $z^{3}$ ) ergab sich, dass diese Annahme nicht stichhaltig war, da das in Anwendung gezogene Jodkali durchaus chemisch rein war.

Für die Anschauung, dass die Zeichen des Jodismus hauptsächlich dann auftreten, wenn Jod im Körper zurückgehalten wird, sprechen die Untersuchungen von Ehlers. ${ }^{4}$ )

In einem längeren Aufsatz besprechen Röhmann und Malachowskis) die Entstehung und Therapie des acuten Jodismus, sie konnten einen Unterschied zwischen kleinen und grossen Dosen von Jodkali auf die Häufigkeit des Auftretens von Jodismus nicht finden.

Ebenfalls für die Annahme, dass der Jodismus hauptsächlich dann hervorgerufen werde, wenn Jod im Körper zurückgehalten werde, sprechen die Beobachtungen von Gerson. ${ }^{\mathbf{b}}$ ) Bei einem Manne mit latenter Nephritis traten schon nach 2 Gr. Jodkali schwere Intoxicationserscheinungen auf. Dass in diesem Falle durch die kranken Nieren Jod nicht ausgeschieden

1) Berl. klin. Wochenschr. 11. 1893.

${ }^{2}$ ) Centralbl. f. klin. Med. 9. 1886.

s) Centralbl. f. klin. Med. 9. 1886.

4) Hosp. Tid. VII. 1. 1889.

5) Therap. Monatsh. VII. 1889.

$\left.{ }^{6}\right)$ Münch. med. Wochenschr. 28. 1889. 
werden konnte, stellte sich durch die Untersuchung des eiweisshaltigen Urins heraus, in welchem Jod nicht nachzuweisen war. Derartige Beobachtungen sind von grosser praktischer Bedeutung gerade für die Behandlung der Psoriasis, man wird bei jedem solchen Falle, bevor man ihn einer Jodcur unterzieht, genau und mehrmals den Urin auf etwaigen Eiweissgehalt prüfen müssen und den Patienten nur bei vollkommenem Freisein des Urins von Albumen einer Jodcur unterwerfen dürfen. Fälle, wie die von Dujardin-Beaumetz, ${ }^{1}$ ) in welchen auf 6.0. Jodkali Intoxicationserscheinungen auftreten, werden sicher nicht zu den besonders bemerkenswerthen Fällen gerechnet werden können. Wenn nun eine Anzahl von Autoren, die ich oben aufgeführt habe, auch bei unverhältnissmässig grossen Dosen Jodkali (30-60 Gr. pro die) keine Intoxicationserscheinungen auftreten sahen, so kann man doch nicht annehmen, dass diese Tag für Tag gegebenen Mengen ebenso rasch wieder ausgeschieden werden, als die mittleren, welche ja vielleicht diuretisch wirken könnten im Sinne: der obigen Ausführung. Zür Erklärung des auffälligen Umstandes, dass diese grossen Dosen nicht toxisch wirken, können wir nicht umhin, nach einem anderen Moment zu suchen, den wir freilich nicht näher definiren können, es ist das die individuelle Toleranz.

Oppenheimer ${ }^{2}$ ) hat ganze Familien kennen gelernt, bei welchen eine Intoleranz gegen Jockali hereditär ist. Ward man aus irgend einem Grunde reranlasst, Jodkali zu verabreichen, so kounte man schon nach 3 Centigrammi die stürmischen Erscheinungen einer gestörten Blutcireulation, Herzklopfen, kleinen Puls, Klopfen der Carotiden, das Gefuihl als ob der Schädel bersten müsse, bei ihnen beobachten.

Wenn bei ganz gesunden Individuen so kleine Dosen schon toxisch wirken, so kann man kaum annehmen, dass sie durch Retention im Organismus diese Wirkung entfalten, hier kann es wohl nur das Jod als solches sein, welches bei seinem Durchgang durch den Organismus diesen in einzelnen seiner Theile entweder Schleimhäute oder Nervensystem in der Weise irritirt, dass die Intoxicationserscheinungen als Symptome dieser

1) Bull. génér. de thér. T. CXIII.

2) Therap. Monatsh. 12. 1889. 
Ueber die Behandl. der Psoriasis mit grossen Dosen von JK. 347

fast specifischen Irritation auftreten. Worauf diese Eigenthümlichkeit einzelner Organismen beruht, entzieht sich vollkommen der Beurtheilung.

Keinenfalls möchte ich die Anschauung Woo d's ${ }^{1}$ ) zu der meinigen machen, dass Personen, welche tolerant gegen grosse Joddosen sind, leichter an Syphilis erkranken als andere, bei denen diese Widerstandsfähigkeit fehlt, geht er ja doch so weit, bei Toleranz gegen. Jod allein aus diesem Symptome syphilitische Infection für wahrscheinlich zu halten. Würde man sich auf diesen Standpunkt stellen, so _hätte man 'eine' einfache Erklärung für die Aetiologie vieler Fälle von $\mathbf{P}_{\text {soriasis, }}$ sie würden eben dann syphilitischer Natur sein, eventuell abgeschwächte Formen hereditärer Spätsyphilis. Dass dem nicht so ist, wenigstens nicht für die von mir beobachteten Fälle, vermag ich mit Sicherheit zu behaupten.

Es erübrigt mir nun noch, die einzelnen Erscheinungen des Jodismus zu besprechen. Die leichtesten Formen des Jodismus, bestehend in Schnupfen, reichlicher Thränensecretion, Kopfschmerzen fehlten fast in keinem meiner Fälle, wenn auch darüber nicht in allen Krankengeschichten eigene Vormerkungen zu finden sind. So lange diese Erscheinungen nicht heftig waren, sah ich mich nicht veranlasst, den Gebrauch von Jodkalium zu unterbrechen, ich habe ebenso wie Haslund gesehen, dass diese geringfügigen Symptome bei langsamém Fortgebrauch des Mittels offenbar in Folge von Gewöhnung an den durch abgespaltenes Jod entstehenden Reizzustand der Schleimhäute bald vollkommen verschwanden. Im ersten Falle musste wegen heftiger Störungen von Seite des Digestionstractus vor vollendeter Cur Jodkalium ganz ausgesetzt und eine locale Behandlung der Psoriasis substituirt werden. So eigenartige nervöse Störungen, wie sie von einzelnen Aútoren berichtet werden, sah ich in keinem meiner Fälle. Kopp ${ }^{2}$ ) beobachtete das Auftreten von eigenthümlichen Schmerzen in den Fusssohlen, Dujardin-Beaumetz ${ }^{3}$ ) Schmerzen in den

1) Brit. med. journ. 1890 .

$\left.{ }^{2}\right)$ Münch. med. W. 1887.

3) $1 . \mathrm{c}$. 
Extremitäten, Hutchinson ${ }^{1}$ ) mitunter dauernde depressive

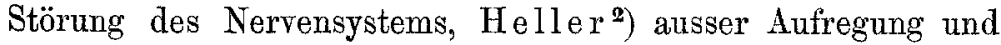
Unruhe, Benommenheit des Sensoriums, Schmerzen in den Fingern. Von Andern sind noch schwerere Erscheinungen von Seite des Nervensystems beobachtet worden, Schwindelanfälle, Gedankenflucht, Schlafsucht, Coma und Delirien s. Lew in ") und Finger ${ }^{4}$ ) (der acute Jodismus und seine Gefahr in der Syphilisbehandlung) und $\mathrm{Haslund}{ }^{5}$ ) Abgestumpftheit, Verwirrtheit, Schwindel. Husemann ${ }^{6}$ ) stellt die ivresse jodique von Lugol, hauptsächlich durch Schwindel and Kopfsehmerz charakterisirt, als nervöses Symptom in Frage.

In den letzten Jahren haben von selteneren Intoxicationserscheinungen die Oedeme des Kehlkopfes Interesse erregt. Derartige Beobachtungen sind an Zahl sehr gering, ich verweise zur näheren Einsichtnahme derselben auf die Zusammenstellungen von Grönouw ${ }^{7}$ ) und von Avellis ${ }^{8}$ ) und führe nur ganz kurz die hieher gehörigen Literaturangaben auf mit Ergänzung aus der neuesten Literatur. Der erste gut beobachtete Fall stammt von $\mathrm{Fenwich}{ }^{9}$ ) aus dem Jahre 1875. In 2 Fällen von Fournier ${ }^{10}$ ) kam ärztliche Hilfe zu spät, in 4 weiteren Fällen konnte der Luftröhrenschnitt rechtzeitig ausgeführt werden. Binz ${ }^{11}$ ) führt Kehlkopfödem bei Jodgebrauch an als aus beim Vorhandensein von Kehlkopfgeschwüren entstehend. Rosenberg ${ }^{12}$ ) veröffentlichte einen Fall von subglottischem Oedem und Grönouw ${ }^{13}$ ) berichtet iiber 4 hieher gehörige Fälle. Weitere 2 Fälle finden sich in dem Lehrbuche von Solis-

1) The practit. June 1891.

2) Wien. med. Presse. 29. 1887.

3) Lehrb. d. Toxikologie.

4) Wien. med. Wochenschr. 1892.

5) 1. c. p. 711 .

6) Specielle Arzneimittellehre p. 788.

7) Therap. Monatsh. III. 1890.

s) Wien. med. Wochenschr. 46-48, 1892.

9) Lancet. 13. 1875.

10) Gaz. d. hôpit. 21. 1889.

11) Vorlesungen über Pharmakologie. 1886 p. 206.

12) Deutsche med. Wochenschr. 1890.

13) l. c. 
Cohen ${ }^{1}$ ) mitgetheilt, den von Baumgarten ${ }^{2}$ ) als Jodödem aufgeführten Fall will Avell is nicht recht als solches gelten lassen. In dem Aufsatz von Avell is ${ }^{3}$ ) findet sich ein von ihm selbst und ein aus der Clientel von Fischenich in Wiesbaden stammender Fall aufgeführt. In dem Falle von Kessler (Petersb. med. Wochenschr., 27. 1891) handelte es sich wahrscheinlich auch um Glottisödem.

Die neueste Publication hierïber haben wir Schmiege$10 \mathrm{w}^{4}$ ) zu verdanken, der zwei Fälle von Jodödem mittheilt, in dem ersten seiner Fälle trat die Larynxstenose ungemein rasch -auf, so dass die Tracheotomie. möglichst rasch mittels eines gewöhnlichen Bistouri ausgeführt werden musste. In dem zweiten Falle gingen die stürmischen Erscheinungen vorüber, ohne dass ein operativer Eingriff nöthig gewesen wäre.

In keinem der von mir beobachteten Fälle wurde ein Oedem des Kehlkopfes beobachtet, dagegen stellte sich in Fall 10 ein intensives Erythem des weichen Gaumens mit ödematöser Anschwellung der Uvula auf, nachdem die tägliche Dosis auf $4^{\circ} 0$ gesteigert worden war. Bei der eigenthümlichen Form des Erythems ohne jede Betheiligung der Tonsillen und bei dem raschen Rückgang der Erscheinungen ist die Annahme, dass es sich um eine toxische (Angina jodica) und nicht um eine infectiöse Form der Angina gehandelt habe, voll berechtigt, wogegen auch nicht das die Angina begleitende Fieber spricht.

Während derartige Erytheme der Mundrachenschleimhaut weniger häufig vorzukommen scheinen, s. Ros e's ${ }^{5}$ ) ersten Fall, sind Erytheme sowohl ohne als mit Exsudationsvorgängen auf der äusseren Haut als ein nicht ungewöhnliches Vorkommen zu bezeichnen. $0^{\prime}$ Reilly ${ }^{6}$ ) beschrieb zuerst eine bullöse Form des Jodexanthems, Pellizari ${ }^{7}$ ) einen papulösen und rupiaförmigen Hautausschlag, dessen Ausbruch Frost und Fieber

1) Diseases of the throat. etc. p. 442.

$\left.{ }^{2}\right)$ Deutsche med. Wochenschr. 9. 1892 und Pest. med. chir. Presse. 78. 1892 .

3) 1. c.

4) Arch. f. Laryngol. I. 1.

5) Virch. Arch. 35, p. 17.

6) New York med. Gaz. Jan. 1854.

7) Lo speriment. 1884 . 
vorausging. Nach Ricord soll eine blutigseröse Infiltration des Bindegewebes vorkommen (s. $\mathrm{Hu}$ semann, Arzneimittellebre). In einem Falle von Elliot ${ }^{1}$ ) traten nach 2 Dosen Jodkalium à 0.25 rothe Flecken im Gesicht auf, welche sich nachher zu. variolaähnlichen Knötchen umwandelten. Ducrey ${ }^{2}$ ) sah zwei Fälle von Jodexanthem, in dem einen handelte es sich um anthraxähnliche Pusteln, in dem anderen um eine hämorrhagische Urticaria, während ein Fall von $W_{0} l^{3}$ ) ein dem Pemphigus ähnliches Exanthem darbot und in dem Falle von Taylor ${ }^{*}$ ) sich discrete Tumoren an Stirn, Wange, Augenlidern und Hals entwickelten.

Balkány $\mathrm{i}^{5}$ ) beobachtete ein bläschenförmiges Exanthem im Gesicht und Erythem am Rumpf und an den unteren Extremitäten; Duhring ${ }^{6}$ ) eine local umschriebene phlegmonöse, durch Jod bedingte Dermatitis, der Fall von Anderson, ${ }^{7}$ ) in welchem es sich um Purpura handelte, endete letal. Morrow, ${ }^{8}$ ) welcher ein bullöses Exanthem sah, weist darauf hin, dass manchmal schon kleine Mengen Jodkali $(0.3 \mathrm{p}$. die) genïgen, um solche Exantheme zu erzeugen. $\mathrm{Zu}$ den oben genannten schweren Erscheinungen von Seiten des Nerrensystems. kamen in den Fällen von Heller ${ }^{9}$ ) und Janovsky ${ }^{10}$ ) Petechien an den Händen. Bradley ${ }^{11}$ ) berichtet über ein dem Erythema nodosum entsprechendes Exanthem, Haslund ${ }^{12}$ ) über ein gleiches Exanthem sowie über eines mit Blaseneruptionen und über ein hämorrhagisches purpuraähnliches Exanthem. Ein bullöses Exanthem wird von Guir ${ }^{13}$ ) nach dem Einnehmen von Jodammonium, ein pustulöser Ausschlag von

) Med. record. 1885.

2) Riv. internaz. di Med. e Chirurg. Nov. 1883.

3) Berl. klin. Wochenschr. 35. 1886.

4) Journ. of cut. and genito-ur. diseas. 1886.

5) Pest. med.-chir. Presse. 30. 1887.

6) Med. and surgery reporter. 1887.

7) Treat. on diseas. of the Skin. Liondon 1887.

8) Monatsh. f. prakt. Dermat. 1887 p. 587.

9) 1. e.

10) Monatsh. f, prakt. Derm. 1886 p. 445.

1') Thèse de Paris. 1887.

12) Hospit. tidende. 7. 1889.

${ }^{13}$ ) Joarn. of cut. and genito-urin. diseases. Vol. VI. 5. 1885. 
de Amicis, ${ }^{1}$ ) Pemphigus von Hallopeau, ${ }^{2}$ ) Erythema nodosum von Lesser ${ }^{3}$ ) berichtet und zwar traten diese Eruptionen nach Jodnatrium schwächer auf als nach Jodkali. In einem Falle von Gerson ${ }^{4}$ ) trat ein masernähnliches Exanthem ohne Temperaturerhöhung und ohne Steigerung der Pulsfrequenz auf, Besnie $\mathbf{r}^{5}$ ) beobachtete Purpura an den Extremitäten und auch $\mathrm{Hutchins}$ on ${ }^{6}$ ) berichtet über Jodexanthem. In einem Falle von $\mathrm{Cutler}^{7}$ ) und in 2 Fällen von Giovanninis) handelte es sich um eine Dermatitis tuberosa. Ein Fall von Mackenzie, ${ }^{9}$ ) der ein jjähriges Kind betraf, endete unter den Erscheinungen einer Purpura hämorrhagica letal. Der von $\mathrm{Kämpfer} \mathrm{r}^{10}$ ) beobachtete sowie der von ihm citirte Fall von Talamon und der von Taylor ${ }^{\mathbf{1}}$ ) mitgetheilte betrafen die tuberöse Form des Exanthems, das letztgenannte besass die grösste Aehnlichkeit mit einem papulösen Syphilid. Broc q ${ }^{12}$ ) zählt neunerlei Arten von Hautausschlägen, welche durch Jod bedingt sein können, auf: 1. diffuses Erythem, 2. Papeln mit Urticaria, 3. Bläschen und Ekzem, 4. Pemphigusblasen, 5. Papeln und Pusteln, 6. Anthraxformen, 7. Petechien, 8. Knötchen und Knotenformen, 9. polymorphes Exanthem. Fis che ${ }^{13}$ ) beschreibt unter anderen Ausschlägen auch ein knötchenförmiges. Exanthem. Eine eigenthümliche Beobachtung konnte T e m p le ${ }^{14}$ ) machen bei einem 60jährigen Manne, dessen weisse Kopf- und Barthaare sich nach 8tägigem Eimmehmen von 3mal täglich 0.6 Jodkalium rosenroth färbten. Ge m ${ }^{15}$ ) berichtet über je

1) Ref. Monatsh. f. prakt. Dermat. 1888 p. 34.

2) L'union méd. 1888.

3) Deutsche med. Wochenschr. 14. 1888.

4) Münch. med. Wochenschr. 25. 1889.

5) Annal. d. dermat. 1889.

6) Arch. of Surgery. 1889.

7) Journ. of cutan. and genit.-urin. diseases. Febr. 1889.

8) Lo sperim. 1889 u. Giorn. ital. dell. mal. ven. e delia pelie. 1889.

9) s. Peltes o hn. Berl. klin. Wochenschr. 43. 1889.

10) Centralbl. f. klin. Medic. 6. 1890.

11) Med. News. 18. Juli 1891.

12) Malad. de la peau. Paris 1890.

13) Wiener med. Wochenschr. 1891.

(4) Monatsh. f. prakt. Dermat. XIII. 11.

15) Annal. d. dermat. 1891. 8-9. 
einen Fall von Erythema articatum und von ekzemähnlicher Eruption und über 2 Fälle von pustulo-ulceröser Dermatitis mit Ausgang in Narbenbildung. In je einem Falle ron Feulard ${ }^{1}$ ) und $\mathrm{Kalb}^{2}$ ) handelte es sich um Pemphigus-Eruptionen, in 2 Fällen von $\mathrm{Hyde}^{3}$ ) um ein bullöses Exanthem. Höning ${ }^{4}$ ) beobachtete ein hämorrhagisches quaddelartiges Exanthem der Haut und Gaumenschleimhaut, Walker ${ }^{5}$ ) und Holsten ${ }^{6}$ ) einen tuberösen, beziehungsweise papulösen Ausschlag, der letztere führte schliesslich zu tiefgehenden Zerstörungen der Cutis. Legrand () berichtet ebenfalls über eine Dermatitis tuberosa nach Jodgebrauch. Welche Art von Exanthem sich in dem von Buzzi und Valerios) mit Jodkali behandelten Fall von Actinomykosis zeigte, konnte ich aus dem mir vorliegenden Referat nicht ersehen. Trapesnikow ${ }^{9}$ ) berichtet über einen Fall von Pemphigus regetans jodicus.

Robinson ${ }^{10}$ ) und Russel ${ }^{11}$ ) boobachteten ein hämorrhagisches purpuraähnlıches Exanthem, in dem letzteren Fall erfolgte der Exitus unter den Erscheinungen der Inanition.

Caspary ${ }^{12}$ ) sah ein inpetiginöses Ekzem im Gesicht und an den Vorderarmen nach Jodkaligebrauch. Eigenthümlich ist die Form und Ausbreitung eines Jodexanthems in einem. von Feibes s ${ }^{13}$ ) mitgetheilten Fall, in welchem sich das Exanthem nahezu ausschliesslich auf die gelähmte Körperhälfte localisirte. Von derartigen Nebenwirkungen des Jodkali habe ich bei meinen Fällen nur einmal ein urticariaähnliches Exanthem beobachtet, das im Ganzen 6 Mal während der Jodcur auftrat;

1) Annal. đ. dermat. 1891.

2) Münch. med. Wochenschr. 11. 1889 p. 190.

3) Journ. of cutan. and vener, diseas. Vol. IV. Heft 12.

$\left.{ }^{4}\right)$ Monatsh. f. prakt. Dermat. XIV. 8.

5) Monatsh. f. prakt. Dermat. XIV. 7.

6) New-York. med. Journ. 23. April 1892.

־) Annal. d. Dermat. 8. 1893.

8) Rif. Med. Juli 1893.

9) Therap. Blätter. 2. 1893.

10) The Lancet. 4. III. 1893.

ii) New-York. med. record. Aug. 1893.

${ }^{12}$ Arch. f. Dermat. XXVI. 1. p. 20.

13) Dermatol. Zeitschrift. I. 3. 
jedesmal unter Temperatursteigerung und beträchtlicher Erhöhung der Pulsfrequenz (Fall 12). Ich habe die ganze Literatur über Jodexanthem aus dem Grunde zusammengestellt, um eine Uebersicht über die verschiedenen Formen der Jodexantheme zu bekommen. Lasse ich die am häufigsten zur Beobachtung gelangende Acne jodica ausser Acht und lege der Uebersicht über die Literatur die Eintheilung von Brocq zu Grunde, so ergeben sich folgende Zahlen:

1. Diffuses Erythem: 5 Mal (Gers on, Lesser, Bradley, Haslund, Elliot),

2. Papeln mit Urticaria: $2 \mathrm{Mal}$ (Gemy, Seifert),

3. Bläschen und Ekzem: 3 Mal (Gemy, Bálkanyi, Caspary),

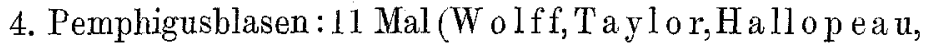
Guire, Haslund, Morrow, Reilly, Kalb, Feulard, Hyde, Tropesnikow),

5. Papeln und Pusteln: $5 \mathrm{Mal}$ (Holsten, Gemy 2. F., de Amicis, Pellizari),

6. Anthraxformen: 1 Mal (Ducrey),

7. Petechien: 9 Mal (Robinson, Russel, Hönig, Mackenzie, Besnier, Haslund, Heller, Andersen, Janorsky),

8, Knötchen und Knotenformen: 9 Mal (Fischer, Talamon, Giovannini, Taylor, Kämpfer, Cutler, Legrand, Walter),

9. Polymorphes-Exanthem (Brocq).

Aus dieser kurzen Zusammenstellung geht herror, dass gerade die schwersten Exanthemformen wie Pemphigus und Petechien zu den häufigsten nach Jodgebrauch entstehenden Hautausschlägen gehören, während die leichteren Formen seltener zur Beobachtung gelangen.

Die Angabe Mor ro's, dass oft schon kleine Dosen Jodkali $(0.3$ pro die) genügen, um derartige Veränderungen auf der Haut hervorzurufen, habe ich weniger an den ron mir beobachteten Fällen als in der Mehrzahl der in der Literatur mitgetheilten Fälle bestätigt gefunden, bei den kurzen Literaturnotizen kommt allerdings dieses Moment nicht genügend zum Ausdruck. 
Es findet dieses eigenthümliche Verhalten der Haut vielleicht seine Erklärung in dem Verhalten des Gefässsystems gegen Jod. Die Einwirkung des Jod auf das Gefässsystem geht sowohl aus dem Thierexperiment als aus klinischen Beobachtungen hervor. Wenn auch $\mathrm{B} \ddot{o} \mathrm{hm}$ und $\mathrm{B}$ e r g $\mathrm{g}^{1}$ ) weder Contraction der Capillaren noch Aenderungen des Blutdruckes nach Jod-oder Jodjodnatrium-Injectionen constatiren konnten, so stehen diesen negativen Resultaten doch eine Reihe von älteren und neueren Beobachtungen gegenïber, nach denen dem Jod ein wesentlicher Einfluss auf das Gefässsystem zukommt. So gibt Rose ${ }^{2}$ ) an, dass es, in grossen Mengen dem Körper einverleibt, einen Gefässkrampf hervorrufe. Aus den Untersuchungen ron Bogole p off $\mathrm{f}^{3}$ ) an Hunden geht hervor, dass Jodkalium (zu $18 \mathrm{Cgr}$.) in die Venen gebracht, rasch eine Dilatation der peripheren Getässe, constantes Abfallen des Blutdruckes bei gesteigerter Pulsfrequenz veursacht. "Von besonderem Interesse sind die experimentellen Studien G. Sée's ${ }^{4}$ ) über die Wirkungsweise des Jodkalium auf das Herz. G. S é e trennt genau die Wirkung des Kalisalzes auf das Herz, auf die Vasoconstriktoren und auf den Blutdruck von der des Jod. Er unterscheidet bei der Jodkaliwirkung auf das Thier (Hund) zwei Phasen in der Wirkung. Die erste entspricht der Wirkung des Alkali, es wird während diese die Herzaction beschleunigt, der Blutdruck in Kurzem um einige Centimeter erhöht, und es bleibt diese Erhöhung für längere Zeit bestehen, nach längerer Zeit - etwa eine Stunde nach der Einspritzung - - beginnt die zweite Phase der Wirkung, welche für Jodkalium und Jodnatrium identisch und wohl als Ausdruck der Jodwirkung anzusehen ist. Der Blutdruck sinkt, die Herzation wird frequenter. Das Abfallen des Blutdruckes dauert mehrere Stunden und sinkt auf ein Minimum, um ganz allmälig wieder emporzusteigen. Die Wirkung auf den Blutdruck erklärt sich aus der Verengerung (Kaliwirkung) resp. Erweiterung der Gefässe (Jodwirkung). Ob man mit Hilfe dieser Thatsachen in der Lage sein wird, die Wirkungen des Mittels

1) Arch. f. exper. Path. V.

2) $1 . \mathrm{c}$.

3) Schmidt's Jahrb. 1877. 1. p. 14.

$\left.{ }^{2}\right)$ Wiener med. Wochenschr. 47. 1889. 
Ueber die Behandl. der Psoriasis mit grossen Dosen von JK. 355

bei Psoriasis und Urticaria ${ }^{1}$ ) zu erklären, ist noch fraglich. Es ruft demnach das Jod überall nicht nur auf den Schleimhäuten, sondern auch auf der äusseren Haut Hyperämien hervor, welche nach grösseren Dosen sogar zu Hämorrhagien (s. oben) Veranlassuug geben können. Diese Gefässdilatation kommt aber nicht durch Lähmung des vasomotorischen Centrums zu Stande, wie nach Chloral oder Amylnitrit, sondern durch Einwirkung auf die Gefässe der Organe oder der oberflächlichen Gewebsschichten.

Die Untersuchungen Trasbots ${ }^{2}$ ) über die Wirkungsweise des Jodkalium bei Thieren weichen wenig von den Ergebnissen G. Sée's ab, während Prevost und Binet ${ }^{3}$ ) keinen besonderen Einfluss des Jods auf den arteriellen Blutdruck und auf die Circulation gefunden haben. $\mathrm{Ob}$ bei ihnen die Beobachtungszeit zu kurz war, vermag ich nicht zu entscheiden, die eigentliche Jodwirkung beginnt ja nach G. Sé e nicht sofort nach der Einverleibung des Jod, sondern erst längere Zeit nachher.

Klinische Beobachtungen über das Verhalten der Circulation gegen Jod sind sehr spärlich, und soweit es sich um die Darreichung von Jodkalium handelt, durchaus nicht einwandfrei, weil hier die Wirkung des Kalisalzes von der des Jod nicht zu unterscheiden ist. Haslund beobachtete nur bei einem seiner Fälle neben nervösen Erscheinungen Herzklopfen, starke Beschleunigung der Herzaction (tumultuarischen Herzschlag) ohne Steigerung der Temperatur. $\mathrm{Ob}$ die von Rieder ${ }^{4}$ ) beobachtete Herzschwäche dem Jod zuzuschreiben ist, vermag ich nicht zu entscheiden. Bradley ${ }^{5}$ fand als constantes Symptom des Jodismus Pulsbeschleunigung ohne Erhöhung der Temparatur, manchmal eher eine Erniedrigung derselben. Dass für das Zustandekommen einer derartigen Jodwirkung auch individuelle Verhältnisse massgebend sind, hebt 0 ppenheimer ${ }^{6}$ ) hervor, der Kenntniss hat ron ganzen

1) Stern. Münch. med. Wochenschr. 1890.

2) Acad. de Médic. Paris 15. Oct. 1889.

3) Revue méd. d. la Suisse rom. 1890. 8.

4) Münch. med. Wochenschr. 5. 1887.

5) Thèse de Paris. 1887.

6) Therap. Monatsh. XII. 1889. 
Familien, bei welchen eine Disposition zu solcher Jodwirkung besteht. Wird man aus irgend einem Grunde veranlasst, Jodkalium zu verabreichen, so konnte man schon nach 0.03 die stürmischsten Erscheinungen einer gestörten Blutcirculation, Herzklopfen, kleinen Puls, Klopfen der Criotiden, das Gefühl als ob der Schädel bersten müsse, bei ihnen beobachten. Eine Hypothese für diese Fälle vermag $O$ pp e nhe i m e r nicht zu geben. Man könnte nur vermuthen, dass bei einer gewissen Disposition des Gefässnervensystems die chemische Zusammensetzung desselben. der Art sei, dass Jodkalium eine Veränderung hervorbringen könne und dass beim Mangel dieser eigenthümlichen Beschaffenheit das Jodkalium nur die Wirkung eines Kaliumsalzes verursache.

Wenn ich nach dieser Betrachtung über die Jod- resp. Jodkaliwirkung meine Fälle durchsehe, so komme ich zu dem Schlusse, dass bei genauer Beobachtung solcher Fälle, bei denen Jodkali in steigender Dosis längere Zeit gegeben wird, nahezu regelmässig ein Einfluss auf die Circulation sich bemerkbar macht, den ich nicht ohne Weiteres als Kaliwirkung auffassen kann. Wenn ich auch nicht überall in den kurzen Krankengeschichten das Verhalten des Pulses hervorgehoben habe, so fehlte doch in keinem der Fälle eine an einzelnen Tagen zu beobachtende Steigerung der Pulsfrequenz. Am lehrreichsten ist der Fall Höfling, bei welchem ohne Erhöhung der Temperatur anfallsweise ausserordentliche Erhöhungen der Pulsfrequenz bis zu 170 zur Beobachtung gelangten (s. d. Curve). Was mir gerade in diesem Falle für eine vorwiegende Jodwirkung zu sprechen scheint, ist der Umstand, dass die jedesmalige Steigerung der Pulsfrequenz erst am Tage nach jedesmaliger Erhöhung der Jodgabe auftrat. Würden solche Steigerungen der Pulsfrequenz als Kaliwirkung anzusehen sein, so muisste nach den Untersuchungen von G. Seé diese Erscheinung kurz nach der erhöhten Jodkalidose, also an dem gleichen Tage zur Erscheinung gekommen sein. Es hatten im Uebrigen diese immerhin recht aufälligen Störungen der Circulation so wenig Einfluss auf das Allgemeinbefinden, dass uns ein Aussetzen des Mittels nicht nöthig erschien. In wie weit bei den Psoriatikern eine Disposition zu solchen Circu- 
lationsstörungen vorliegt, vermag ich noch nicht zu entscheiden, da ich erst in letzter Zeit einen meiner Zuhörer, Herrn D e hnik e beauftragt habe, die von $\mathrm{Pol}$ ote $\mathrm{b}$ n off $\mathrm{f}^{\mathbf{1}}$ ) gemachten Angaben über die Erregbarkeit des Herzens bei Psoriatikern zu prüfen. Bei Individuen mit so leicht erregbarem Herzen und hohem Erethismus des Gefässsystems, wie in den ron 0 p penheimer erwähnten Fällen möchte freilich der Fortgebrauch so hoher Dosen (20-22 Gr.) Jodkalium nicht rathsam erscheinen.

Einer anderen ebenfalls interessanten Wirkung des Jodkalium habe ich noch zu gedenken, nämlich der Erzeugung von Fieber.

Schon bei $\mathrm{Husemann}{ }^{2}$ ) findet sich eine Notiz, aus der hervorgeht, dass Jod ausser Pulsbeschleunigung auch Temperaturerhöhung hervorrufen kann, die hinsichtlich ihrer Intensität und Dauer zur localen Einwirkung in keinem Verhältnisse steht. Auch Rose beobachtete Steigerung der Körpertemperatur. Bogolopeff ${ }^{3}$ ) fand bei seinen Thierversuchen, dass die Temperatur öfter um einige Grade ansteigt, was ebenso wie die Abnahme des Blutdruckes mit Erweiterung der peripheren Gefässe zusammenfällt. Beobachtungen, wie die von Jan ow sky, ${ }^{4}$ ) von $\mathrm{Kämpfer}{ }^{5}$ ) und Seifert (Fall 12), wonach das Auftreten von Exanthemen von Temperaturerhöhungen begleitet, resp. mit solchen eingeleitet werden kann, können nicht als reines Jodfieber bezeichnet werden.

Malachowski ${ }^{6}$ ) bezeichnet die beiden ron ihm beobachteten Fälle als die ersten in der Literatur mitgetheilten, in welchen ohne Zweifel das Fieber als eine reine Jodwirkung anzusehen war. Malachowski schliesst dies daraus, weil 1. das Fieber kurze Zeit nach Beginn der Jodtherapie bei vorher fieberlos erkrankten Individuen auftrat, weil 2. das Fieber in kurzer Zeit nach Aussetzen des Mittels verschwand and weil 3. trotz sorgfältiger Untersuchung nirgends am ganzen Körper

\footnotetext{
1) Dermat. Studien. 1890.

2) Handbuch d. Fonikologie. 1862.

3) 1 . c.

4) Monatsh. f. prakt. Dermat. 1886 p. 445.

5) 1 . c.

6) Therap. Monatsh. IV. 1889.
} 
sich ein Anhaltspunkt für das Fieber bot und auch keinerlei subjective Beschwerden auf eine Fieberquelle hinwiesen. Der Puls hatte in beiden Fällen eine dem Fieber entsprechende Vermehrung gehabt.

Eine Erklärung für das Fieber vermag Malachowski nicht zu geben, er weist nur hin auf die Möglichkeit einer eventuellen Beeinflussung thermischer Centren im Gehirn.

Diesen Beobachtungen von Malachowsky lassen sich meine 11 Fälle der II. und III. Gruppe anreihen. Während in den ersten beiden Fällen die Körpertemperatur während der ganzen Dauer der Behandlung normal geblieben war, zeigte nur die Pulsfrequenz an einzelnen Tagen eine mässige Erhöhung zwischen 100 und 128 ohne Herzklopfen und ohne Störung des Allgemeinbefindens, so dass mit der Jodmedication auszusetzen unnöthig erschien.

Von den Fällen der II. und Gruppe III. muss ich von vorneherein, um etwaigen Einwänden zu entgehen, Fall 3, 4 und 5 sowie Fall 10 und 12 ausscheiden, weil es sich hier nicht um reines Jodfieber, sondern um anderweitige Intoxicationserscheinungen handelte, in deren Begleitung das Fieber zur Beobachtung kam.

In Fall 4 trat schon am 3. Tage nach der ersten Dosis eine ziemlich starke Jodacne auf und in deren Begleitung eine mehrere Tage lang dauernde Temperatursteigerung mit mässiger Pulsbeschleunigung (s. Curve). Die rerhältnissmässig lange Dauer des Fiebers und die geringe Beschleunigung der Pulsfrequenz würden auch abgesehen von der Jodacne gegen die Bedeutung dieses Fiebers als Jodfieber sprechen können.

Auch die Erscheinungen in Fall 3 und 5 möchte ich nicht ohne Weiteres als Jodfieber auffassen, da sich leichte Temperaturerhöhungen (37.8-38.0) im Anschluss an Jodschnupfen und heftige Kopfschmerzen bemerkbar machten. Auffallend ist allerdings die im. Verhältniss zur Körpertemperatur erhebliche Beschleunigung der Pulsfrequenz (116-120), welche in dem vorher bezeichneten Falle bei einer Temperatur von $39 \cdot 0$ nur 96 betragen hatte.

Trotzdem will ich, um scharfe Kritik zu üben, auch diesen Fall nicht unter die reinen Jodfieber rubriciren, sondern 
Ueber die Behandl. der Psoriasis mit grossen Dosen von JK. 359

annehmen, dass der starke Schnupfen zu der Temperatursteigerung Veranlassung gegeben hat.

Bei Fall 12 erklärt das über den ganzen Körper sich ausbreitende urticariaähnliche Exanthem das Auftreten der Temperatursteigerung, doch zeigte sich auch hier wieder wie in Fall 5 eine mit der Temperatursteigerung in keinem Verhältniss stehende Beschleunigung der Herzaction.

Der Fall 10 ist nur zum Theil aus der Betrachtung auszuschliessen, indem sich in den ersten Tagen der Jodbehandlung Fieber an ein Erythem des Rachens mit ödemalöser Anschwellung der Uvula anschloss, resp. diese Erscheinungen begleitete.

Dagegen kann die in der zweiten Hälfte der Behandlungszeit zur Beobachtung gelangte Erhöhung der Körpertemperatur und der Pulsfrequenz als Jodfieber bezeichnet werden aus gleich zu besprechenden Gründen.

In den übrigen 6 Fällen handelte es sich unzweifelhaft um Fiebererscheinungen, welche direct auf die Jodeinwirkung zu beziehen sind. Für dieses Jodfieber möchte ich als Paradigma die in Fall 10 während der zweiten Hälfte der Behandlungszeit auftretenden Erscheinungen bezeichnen. Es findet sich hier auf der zweiten Hälfte der Curve eine plötzliche Steigerung der Temperatur und Beschleunigung der Pulsfrequenz angegeben, letztere der Temperatursteigerung um nahezu 24 Stunden vorausgehend. Nach 12 Stunden Abfall der Temperatur auf resp. etwas unter die Norm, während die Pulsfrequenz noch Tage lang unverhältnissmässig hoch bleibt. Am zweiten Tage nochmals geringe Temperatursteigerung bei gleichbleibender Pulsfrequenz.

Ein ähnliches Verhalten weist auch Fall 6 auf, doch zeigte sich bei diesem die Beschleunigung der Pulsfrequenz bei Weitem nicht so auffällig wie bei Fall 10.

Wenn man die Krankengeschichten und die Curven genauer betrachtet, so findet man, dass solche Anfälle von Jodfieber sowohl bei grossen als bei kleinen Dosen auftreten können. Von besonderem Interesse scheint mir noch der Fall $8 \mathrm{zu}$ sein, bei welchem jede Erhöhung der Tagesdosis Jodkali von einer Erhöhung der Temperatur und der Pulsfrequenz gefolgt war. Ich bin der Ansicht, dass gerade solche Beobachtungen am 
besten geeignet sind, um die Abhängigkeit des Fiebers von der Jodwirkung klar zu stellen.

Zur Charakteristik des Jodfiebers, die uns Malachowski gibt, hätte ich nach meinen Beobachtungen nur wenig hinzuzufügen, nur einige Bemerkungen glaube ich noch anschliessen zu sollen.

Malachowski gibt an, dass der Puls in seinen beiden Fällen eine dem Fieber entsprechende Vermehrung gehabt habe. Aus meinen Beobachtungen darf ich wohl den Schluss ziehen, dass gerade das Missverhältniss zwischen Temperatursteigerung und Erhöhung der Pulsfrequenz dazu beiträgt, um ausser den von Malachowski genannten Momenten das Jodfieber als solches zu charakterisiren, es braucht dieses Missverhältniss nicht regelmässig vorhanden zu sein, dürfte aber doch in der Mehrzahl der Fälle zur Beobachtung gelangen und gerade jene Fälle scheinen mir besonders bemerkenswerth, in welchen die Erhöhung der Pulsfrequenz nicht mit dem Eintritt der Temperatursteigerung zusammenfällt, sondern dieser um 12-24 Stunden vorausgeht.

Ferner hat Malachowski angegeben, dass das Jodfieber in kurzer Zeit nach Anssetzen des Mittels rerschwindet, Fall 8 liefert den Beweis, dass das Fieber auch zurückgeht, wenn der Organismus sich an jene Höhe der Jodkalidosis gewöhnt, welche die Fiebererscheinungen hervorgerufen hat, so dass man also bei leichtem Jodfieber nicht gezwungen ist, mit dem Medicament auszusetzen.

Und zum Schluss möchte ich mir noch eine Bemerkung erlauben. Man wird in der Hospitalbehandlung bei fortgesetzter Jodmedication in steigender Dosis, sobald man nur ganz regelmässige exacte Temperaturbestimmungen und Zählungen der Pulsfrequenz vornimmt, leichtere Grade von Jodfieber öfters beobachten. Die leichteren Grade des Jodfiebers werden sicher ohne solche fortlaufende Untersuchungen übersehen, da sie keinerlei Störungen des Allgemeinbefindens bedingen.

Wenn auch meine Untersuchungen und Beobachtungen nicht viel Neues gebracht haben, so schienen sie mir doch der Mittheilung werth. 